\title{
Mathematical analysis and optimal control interventions for sex structured syphilis model with three stages of infection and loss of immunity
}

\author{
Abdulfatai Atte Momoh ${ }^{1 *}$ (D, Yusuf Bala ${ }^{1}$, Dekera Jacob Washachi ${ }^{1}$ and Dione Déthié ${ }^{2}$
}

"Correspondence:

abdulfatai@mautech.edu.ng

1 Department of Mathematics,

Modibbo Adama University of

Technology, Yola, Nigeria

Full list of author information is

available at the end of the article

\section{를 Springer}

\begin{abstract}
In this study, we develop a nonlinear ordinary differential equation to study the dynamics of syphilis transmission incorporating controls, namely prevention and treatment of the infected males and females. We obtain syphilis-free equilibrium (SFE) and syphilis-present equilibrium (SPE). We obtain the basic reproduction number, which can be used to control the transmission of the disease, and thus establish the conditions for local and global stability of the syphilis-free equilibrium. The stability results show that the model is locally asymptotically stable if the Routh-Hurwitz criteria are satisfied and globally asymptotically stable. The bifurcation analysis result reveals that the model exhibits backward bifurcation. We adopted Pontryagin's maximum principle to determine the optimality system for the syphilis model, which was solved numerically to show that syphilis transmission can be optimally best control using a combination of condoms usage and treatment in the primary stage of infection in both infected male and female populations.
\end{abstract}

MSC: 92D40; 93A30

Keywords: Stability; Hamiltonian; Transmission; Equilibrium states; Epidemiology; Invariant region

\section{Introduction}

Syphilis is one of the infectious diseases, most commonly caused by sexual contact. Spirochete Treponema pallidum is a spiral-shaped bacteria that causes syphilis [23]. When a person contracts syphilis, he or she develops sores, blisters, or ulcers on his genitals anus (bottom), or mouth [2]. The disease is largely transmitted from one person to another when a susceptible person has sex without condoms or shares sex toys with an infected person [33]. Even though the disease is spread from sores, most of those sores disappeared without being recognized [33]. There are three different stages in syphilis disease: primary stage, secondary stage, and latent stage. At the primary stage the sign may appear as a solitary, painless chancre at the site of inoculation. However, the primary chancre may disappear without being noticed by infected persons. If the disease is left untreated at the

(c) The Author(s) 2021. This article is licensed under a Creative Commons Attribution 4.0 International License, which permits use sharing, adaptation, distribution and reproduction in any medium or format, as long as you give appropriate credit to the original author(s) and the source, provide a link to the Creative Commons licence, and indicate if changes were made. The images or other third party material in this article are included in the article's Creative Commons licence, unless indicated otherwise in a credit line to the material. If material is not included in the article's Creative Commons licence and your intended use is not permitted by statutory regulation or exceeds the permitted use, you will need to obtain permission directly from the copyright holder. To view a copy of this licence, visit http://creativecommons.org/licenses/by/4.0/. 
primary stage, then it would advance to the secondary stage. The symptoms at this stage are more visible. It includes mucocutaneous lesions affecting both skins, mucous membranes, and lymph nodes [44]. Mostly, at the secondary stage, there are usually rashes on the palms and sole of an infected person, and it can imitate other infectious and noninfectious conditions. It is important to state that the rashes on the palm and foot of an infected person at secondary syphilis stage may also disappear even without treatment. If the disease is left untreated, then the infected person progresses to the latent stage [44]. The latent syphilis is asymptotic, described by positive syphilis serology without any clinical manifestations [44]. At this stage, syphilis is often categorized in to two parts, early latent syphilis and late latent syphilis. The early latent syphilis is characterized by an infection, which is less than two years, whereas the late latent syphilis, on the other hand, is an infection of the disease for two years or beyond [44]. Transmission of syphilis occurs during primary, secondary, or early latent syphilis; moreover, mother-to-child transmission has been observed after several years of infection with syphilis, especially in an untreated cases [44]. According to World Health Organization (WHO), an estimates of 36.4 million syphilis infection cases were recorded worldwide [43]. About $90 \%$ of new syphilis cases are found in resource-limited countries out of an estimated population of over 12 million new syphilis infections recorded every year in the world [13, 14]. WHO reported that there are 3.4 million annual new cases of syphilis in the African region among people aged 15-49 years [43]. The global incidence rate of syphilis was 1.5 cases per 1000 females and 1.5 per 1000 males in 2012. It is estimated that the global prevalence of syphilis is at $0.5 \%$ among females and $0.5 \%$ among males aged 15-49 years, with the highest prevalence in the WHO African Region [44]. In Nigeria the prevalence of syphilis is estimated to be between 1.3 million and 2.8 million $(0.7-1.5 \%)$ [17,39]. There were 6498 deaths as a result of syphilis infection, 4149 males and 2349 females in the United States of America from 1968 to 2015. Adults now rarely die due to syphilis [31]. About 492,000 infants die each year due to syphilis infection from congenital syphilis in sub-Saharan Africa [30]. In Nigeria, neonatal death rate per 1000 is estimated at 34.1 [21]. Despite the introduction of penicillin for almost over a decade, syphilis continues to be a disease of concern, and therefore the optimal management of syphilis continues to be a controversial topic [12, 40]. Presently, clinical guidelines suggest similar treatment regimens for various stages of syphilis. Penicillin continues to be the drug of choice to treat all stages of syphilis in all populations with tetracycline and cephalosporin acceptable alternate agents for some stages in nonpregnant person $[12,40]$. Penicillin G remains the most suitable drug for treating infected persons with syphilis in all the stages. The stage and clinical manifestations of syphilis dictate the preparation used (i.e., benzathine, aqueous procaine, or aqueous crystalline), dosage, and length of treatment [11]. Persons with latent syphilis of unknown duration require longer treatment duration to ensure that those who did not acquire syphilis within the preceding period are adequately treated [11]. Novel and effective prevention strategies are keenly required to combat syphilis infection because currently there is no vaccine to prevent infection with syphilis [40]. Syphilis is a preventable and potentially eradicable disease [18]. Transmission is prevented, and subsequent new infections are also blocked when syphilis is treated, and hence it reduces the prevalence of syphilis in a population to a minimal level. Mathematical models play a very vital role in the study of the dynamic of infectious diseases; for example, [15] developed and analyzed a mathematical model that includes the basic stages of the disease and assumed that infected individuals acquire temporary 
immunity only after recovery from the latent and tertiary infections. [23] considered a SIR mathematical model to study the effect of presence of partial immunity and vaccine against syphilis infection in a population. The results reveal that health education leading to enhanced biological and behavioral protection against infection and the development of effective vaccine is the most effective way to control syphilis transmission in a highrisk population. [17] presented a new multistage deterministic model for the transmission dynamics of syphilis to qualitatively assess the role of loss of transitory immunity in the transmission process. They show that loss of transitory (natural) immunity can induce the phenomenon of backward bifurcation. [37] proposed a model for the transmission of syphilis in an MSM population that includes infection stages and treatment. [29] used a nonlinear mathematical model to study the dynamics of the spread of syphilis in heterogeneous settings with complications, and two stages of (primary and secondary stages) infection only were considered. [2] formulated a compartmental model to investigate the dynamics of the spread of syphilis in a sexually active population with some measure of disease control. The model undergoes the phenomenon of backward bifurcation and proposed that effective treatment strategies of syphilis in its primary and secondary infected individuals will help reduce the cases. In [26] an investigation was carried out to determine the synergistic interaction between HIV and syphilis using a mathematical model. The paper assessed the impact of syphilis treatment on the dynamics of syphilis and HIV coinfection in a human population where HIV treatment is not readily available or accessible to HIV infected individuals, and they proposed that if a concerted effort is exerted in the treatment of primary and secondary syphilis (in both singly and dually infected individuals), especially with high treatment rates for primary syphilis, then it will result in a reduction in the incidence of HIV (and its coinfection with syphilis) in the population.

However, until now, very few studies were conducted to investigate the optimal control strategies with the view to come up with best way of curtailing the spread of syphilis within a population. [38] incorporated in an epidemiological model for the transmission dynamics of syphilis a control variable to assess the effects of resistance strategies against the disease. He emphasizes the need to reevaluate the current control programs; the development of an effective vaccine associated with health education could be the best way to control syphilis in high-risk populations. [1] formulated and analyzed the dynamics of a syphilis model with the introduction of two controls, and the two time-independent controls represent strategies for improvement of the treatment and cure of the syphilis disease.

[34] used an epidemiological SEIR (Susceptible, Exposed, Infectious, Removed) type model for rubella epidemic via classical and fractional-order Caputo differential operators assuming the periodic transmission rate $\beta(t)$. [35] investigated the dynamics of measles infection with the help of mathematical operators called conformable derivatives of order $\alpha$ (the local derivative index) in the sense of Liouville-Caputo operator of order $\beta$ (the iterated or fractionalizing index). An epidemiological model related with diarrhea transmission dynamics that occurred in Ghana during 2008-2018 was investigated by [36]. The epidemiological model was designed for the very first time with newly devised fractional Caputo-type operator having the fractional order $\alpha$ and the fractal dimension $\tau$. [25] proposed a fractional-order epidemic model with two different operators called the classical Caputo operator and the Atangana-Baleanu-Caputo operator for the transmission of Covid-19 epidemic. The reproduction number $\mathcal{R}_{0}$ was obtained for the prediction and 
persistence of the disease in a population. [5] presented face masks simple but powerful weapons to protect individuals against Covid-19 spread. The focus of their research was to depict the transport of Covid-19 spread through wind with high speed. The stability of nonmonotone critical waves by antiweighted method for a kind of nonmonotone timedelayed reaction-diffusion equations, including Nicholson's blowflies equation, which describes the population dynamics of a single species with age structure, was studied in [45]. In [6], homotopy transform methods, namely, homotopy analysis transform method and homotopy perturbation Sumudu transform method, were implemented to examine the fractional model for HIV infection of $C D 4^{+} T$ lymphocyte cells. A mathematical model for the spread of Covid-19 was analyzed in [3] using theory of stability and optimal control. The proposed model was extended to the concept of nonlocal operators, in which the positiveness of the system solutions were established. [4] presented a detailed analysis of an important class of differential equations called stochastic equations with the new classes of differential operators with global derivatives of integer and noninteger orders. In an attempt to show the applicability of the operators, three epidemiological problems, namely, Zombie virus spread model, the Zika virus spread model, and Ebola model, were studied, and their results showed that more complex real-world problems could be depicted using the classes of differential equations studied.

In this research, we complement and extend the work of [29] by taking into cognizance the standard incidence rate and three stages of infection, namely, primary, secondary, and latent stages of infection of the disease transmission against two stages (primary and secondary) considered by [29], and according to recent revelations of various studies conducted by different authors, the early latent stage can also transmit the disease. However, we have also incorporated an optimal control by using three control strategies consisting of prevention and treatment to investigate the best control strategy that would assist in curtailing the spread of syphilis in a population based on the following assumptions: The latent stage of infection can also transmit the disease, individuals recovered from syphilis infection can also contact the disease after the loss of immunity, the population of both male and female are assumed to be sexually active in all stages of infection, individuals with syphilis in the primary stage can also transmit the disease, and individuals in the susceptible population can be infected with syphilis after having contact with either those in the primary, secondary, or individual at the latent stages of infection.

The paper is presented sectionwise as follows: The syphilis model is formulated in Sect. 2. Mathematical analysis of the syphilis model is presented in Sect. 3. The optimal control problem and analysis of the control problem are presented in Sect. 4. Numerical results and discussion are provided in Sect. 5. Finally, we conclude in Sect. 6.

\section{Formulation of syphilis model}

The total population at time $t$ represented as $N(t)$ is subdivided into smaller classes: susceptible males $S_{m}(t)$, susceptible females $S_{f}(t)$, males with primary stage syphilis infection $I_{m p}(t)$, females with primary stage syphilis infection $I_{f p}(t)$, males with secondary stage syphilis infection $I_{m s}(t)$, females with secondary stage syphilis infection $I_{f_{s}}(t)$, males with latent stage syphilis infection $L_{m}(t)$, females with latent stage syphilis infection $L_{f}(t)$, recovered males $R_{m}(t)$, and recovered females $R_{f}(t)$. So

$$
N(t)=S_{m}(t)+S_{f}(t)+I_{m p}(t)+I_{f p}(t)+I_{m s}(t)+I_{f s}(t)+L_{m}(t)+L_{f}(t)+R_{m}(t)+R_{f}(t) .
$$


Recruitment into the susceptible males at time $t, S_{m}(t)$ is done by the increase in the number of sexually active individuals, who do not previously contact syphilis at rate $\pi_{m}$. The population is also increased by rate $\varphi_{m}$ of males who recovered from syphilis infection after the loss of immunity. The population of susceptible males reduces due to the development of newly infected males with syphilis who progress to the males with primary stage syphilis by a function $\alpha_{f} \psi\left(\frac{I_{f p}+I_{s}+L_{f}}{N}\right) S_{m}$, where $\alpha_{f}$ represents the transmission probability of syphilis females, and $\psi$ is the average number of sexual partners per unit time. The susceptible males reduce as a result of natural driven death at rate $\mu$. So the equation becomes

$$
\frac{d S_{m}}{d t}=\pi_{m}+\varphi_{m} R_{m}-\alpha_{f} \psi\left(\frac{I_{f p}+I_{f s}+L_{f}}{N}\right) S_{m}-\mu S_{m}
$$

Recruitment into the susceptible females at time $t, S_{f}(t)$, is due to an increase in the number of sexually active females who did not previously contact syphilis at rate $\pi_{f}$. Recovery from syphilis infection and later loss of immunity also contribute to increasing in susceptible females at rate $\varphi_{m}$. This population is reduced by acquiring syphilis infection at the quantity $\alpha_{m} \psi\left(\frac{I_{m p}+I_{m s}+L_{m}}{N}\right) S_{f}$ and the migration of such a population to the females with syphilis at the primary stage of infection, where $\alpha_{m}$ is the transmission probability of syphilis infected male. The population is also reduced by death due to the natural factor at rate $\mu$. Thus

$$
\frac{d S_{f}}{d t}=\pi_{f}+\varphi_{f} R_{f}-\alpha_{m} \psi\left(\frac{I_{m p}+I_{m s}+L_{m}}{N}\right) S_{f}-\mu S_{f} .
$$

The population of males with primary stage syphilis at time $t, I_{m p}(t)$, increases due to progression of newly infected syphilis individuals from the susceptible male at the quantity $\alpha_{f} \psi\left(\frac{I_{f p}+I_{s}+L_{f}}{N}\right) S_{m}$ and is reduced due to movement to males with secondary stage syphilis $I_{m s}$ at rate $\gamma_{m}$. This population is further decreased due to natural death at rate $\mu$ and presence of treatment using antibiotics at rate $\sigma_{m_{1}}$, so that

$$
\frac{d I_{m p}}{d t}=\alpha_{f} \psi\left(\frac{I_{f p}+I_{f s}+L_{f}}{N}\right) S_{m}-\gamma_{m} I_{m p}-\mu I_{m p}-\sigma_{m 1} I_{m p}
$$

The population of females with primary stage syphilis at time $t, I_{f p}(t)$, increases as a result of progression of newly infected females with syphilis infection from susceptible female at the quantity $\alpha_{m} \psi\left(\frac{I_{m p}+I_{m s}+L_{m}}{N}\right) S_{f}$ and is reduced due to progression to infected females with secondary stage syphilis $\left(I_{f_{s}}\right)$ at rate $\gamma_{f}$. There is a decrease in the population due to natural death at rate $\mu$ and treatment using antibiotics at rate $\rho_{f_{1}}$. Thus

$$
\frac{d I_{f p}}{d t}=\alpha_{m} \psi\left(\frac{I_{m p}+I_{m s}+L_{m}}{N}\right) S_{f}-\gamma_{f} I_{f} p-\mu I_{f} p-\rho_{f_{1}} I_{f p} .
$$

The infected males with secondary stage syphilis infection at time $t, I_{m s}(t)$, increase due to progression of males with primary stage syphilis $\left(I_{m p}\right)$ to males with secondary stage syphilis $\left(I_{m s}\right)$ at rate $\gamma_{m}$. The population is reduced by the progression of males with secondary stage syphilis $\left(I_{m s}\right)$ at rate $\beta_{m}$ to males with latent stage syphilis $\left(L_{m}\right)$. The population is further reduced by death from natural factor at rate $\mu$ and due to the presence of 
treatment (antibiotics) at rate $\sigma_{m_{2}}$, so that the equation is given by

$$
\frac{d I_{m s}}{d t}=\gamma_{m} I_{m} p-\beta_{m} I_{m s}-\sigma_{m_{2}} I_{m s}-\mu I_{m s}
$$

Females with secondary stage syphilis population of at time $t, I_{f s}(t)$, increase due to progression of females with primary stage syphilis $\left(I_{f_{p}}\right)$ to females with secondary stage syphilis $\left(I_{f_{s}}\right)$ at rate $\gamma_{f}$. The population is reduced by the progression of females with secondary stage syphilis $\left(I_{f s}\right)$ at rate $\beta_{f}$ to the latent stage syphilis $\left(L_{f}\right)$ and is further reduced by a natural death at rate $\mu$ and due to treatment (antibiotics) at rate $\rho_{f 2}$, so that the equation is given by

$$
\frac{d I_{f_{s}}}{d t}=\gamma_{f} I_{f} p-\beta_{f} I_{f s}-\rho_{f_{2}} I_{f s}-\mu I_{f s} .
$$

The population of males with latent syphilis at time $t, L_{m}(t)$, is increased by the progression of infected males with secondary stage syphilis $\left(I_{m s}\right)$ at rate $\beta_{m}$ to the population of males with latent stage syphilis $\left(L_{m}\right)$ and is reduced by a natural death at rate $\mu$. This population is further reduced due to the presence of treatment (antibiotics) at rate $\sigma_{m_{3}}$, so that the equation is given by

$$
\frac{d L_{m}}{d t}=\beta_{m} I_{m s}-\mu L_{m}-\sigma_{m_{3}} L_{m} .
$$

The population of females with latent stage syphilis at time $t, L_{f}(t)$, is increased by the progression of infected females with secondary stage syphilis $\left(I_{f_{s}}\right)$ at rate $\beta_{f}$ to the population of females with latent stage syphilis $\left(L_{f}\right)$ and is reduced by natural death at rate $\mu$. This population is further reduced due to the presence of treatment (antibiotics) at rate $\rho_{f_{3}}$, so that

$$
\frac{d L_{f}}{d t}=\beta_{f} I_{f_{s}}-\mu L_{f}-\rho_{f_{3}} L_{f} .
$$

The population of recovered males at time $t, R_{m}(t)$, is increased due to progression of treated males from primary, secondary, and latent stages of syphilis $\left(I_{m p}, I_{m s}, L_{m}\right)$, respectively, at rates $\sigma_{m_{1}}, \sigma_{m_{2}}$, and $\sigma_{m_{3}}$, which are the treatment rates of syphilis infection in male population. This population is reduced due to natural death at rate $\mu$ and is further decreased due to loss of immunity acquired as a result of treatment and transfer of such individuals to susceptible male population at rate $\varphi_{m}$, so that

$$
\frac{d R_{m}}{d t}=\sigma_{m_{1}} I_{m p}+\sigma_{m_{2}} I_{m s}+\sigma_{m_{3}} L_{m}-\mu R_{m}-\varphi_{m} R_{m} .
$$

The population of recovered females at time $t, R_{f}(t)$, is increased due to progression of treated females from primary, secondary, and latent stages of syphilis infections $\left(I_{f p}, I_{f s}, L_{f}\right)$, respectively, at rates $\rho_{f_{1}}, \rho_{f_{2}}$, and $\rho_{f_{3}}$. This population is reduced due to natural death at rate $\mu$ and is further decreased due to loss of immunity acquired as a result of treatment and transfer of such an individual to the susceptible male population at rate $\varphi_{f}$ :

$$
\frac{d R_{f}}{d t}=\rho_{f_{1}} I_{f p}+\rho_{f_{2}} I_{f_{s}}+\rho_{f_{3}} L_{f}-\mu R_{f}-\varphi_{f} R_{f} .
$$

The model diagram is presented in Figure 1. 


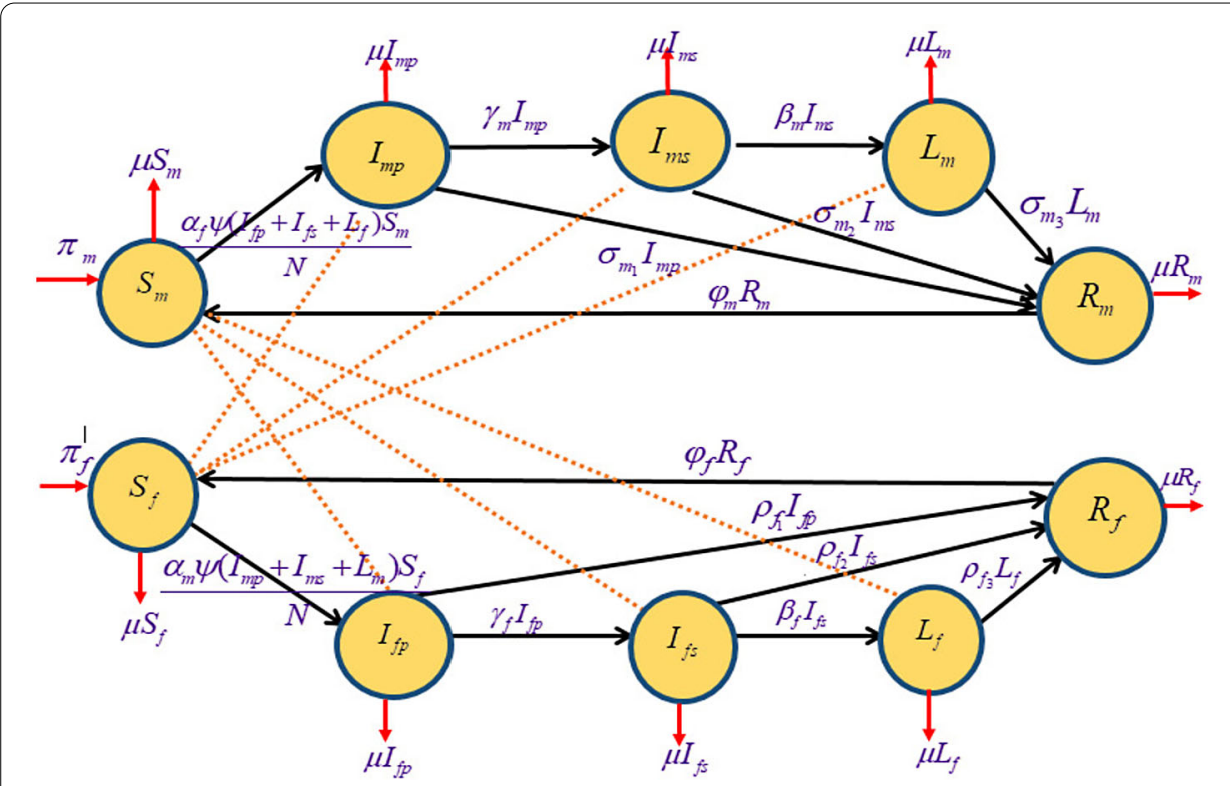

Figure 1 Model with three stages of infection and three control interventions

Therefore we present the syphilis model with three stages of infection:

$$
\left\{\begin{array}{l}
\frac{d S_{m}}{d t}=\pi_{m}+\varphi_{m} R_{m}-\alpha_{f} \psi\left(\frac{I_{f p}+I_{f s}+L_{f}}{N}\right) S_{m}-\mu S_{m}, \\
\frac{d I_{m p}}{d t}=\alpha_{f} \psi\left(\frac{I_{f p}+I_{s}+L_{f}}{N}\right) S_{m}-\gamma_{m} I_{m p}-\mu I_{m p}-\sigma_{m 1} I_{m p}, \\
\frac{d I_{m s}}{d t}=\gamma_{m} I_{m} p-\beta_{m} I_{m s}-\sigma_{m_{2}} I_{m s}-\mu I_{m s}, \\
\frac{d L_{m}}{d t}=\beta_{m} I_{m s}-\mu L_{m}-\sigma_{m_{3}} L_{m}, \\
\frac{d R_{m}}{d t}=\sigma_{m_{1}} I_{m p}+\sigma_{m_{2}} I_{m s}+\sigma_{m 3} L_{m}-\mu R_{m}-\varphi_{m} R_{m}, \\
\frac{d S_{f}}{d t}=\pi_{f}+\varphi_{f} R_{f}-\alpha_{m} \psi\left(\frac{I_{m p}+I_{m s}+L_{m}}{N}\right) S_{f}-\mu S_{f}, \\
\frac{d I_{f p}}{d t}=\alpha_{m} \psi\left(\frac{I_{m p}+I_{m s}+L_{m}}{N}\right) S_{f}-\gamma_{f} I_{f} p-\mu I_{f} p-\rho_{f_{1}} I_{f p}, \\
\frac{d I_{s s}}{d t}=\gamma_{f} I_{f} p-\beta_{f} I_{f_{s}}-\rho_{f_{2}} I_{f_{s}}-\mu I_{f s}, \\
\frac{d L_{f}}{d t}=\beta_{f} I_{f s}-\mu L_{f}-\rho_{f_{3}} L_{f}, \\
\frac{d R_{f}}{d t}=\rho_{f_{1}} I_{f p}+\rho_{f_{2}} I_{f_{s}}+\rho_{f_{3}} L_{f}-\mu R_{f}-\varphi_{f} R_{f},
\end{array}\right.
$$

with the initial conditions

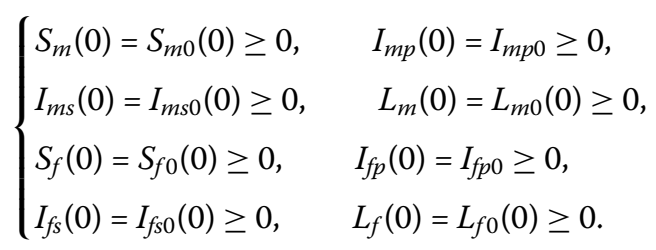

The associated subclasses are added to get the dynamics of the total population of system (2), which yields

$$
\frac{d N}{d t}=\pi_{m}+\pi_{f}-\mu N
$$


Table 1 Syphilis model variables

\begin{tabular}{ll}
\hline Variable & Description \\
\hline$S_{m}(t)$ & Susceptible males at time $t$ \\
$I_{m p}(t)$ & Males with primary stage syphilis at time $t$ \\
$I_{m s}(t)$ & Males with secondary stage syphilis at time $t$ \\
$L_{m}(t)$ & Males with latent stage syphilis at time $t$ \\
$R_{m}(t)$ & Recovered males from syphilis at time $t$ \\
$S_{f}(t)$ & Susceptible females at time $t$ \\
$I_{f p}(t)$ & Females with primary stage syphilis at time $t$ \\
$I_{f s}(t)$ & Females with secondary stage syphilis at time $t$ \\
$L_{f}(t)$ & Females with latent stage syphilis at time $t$ \\
$R_{f}(t)$ & Recovered females from syphilis at time $t$ \\
\hline
\end{tabular}

Table 2 Syphilis model parameters notation and values

\begin{tabular}{|c|c|c|c|}
\hline Parameters & Description & Values & Source \\
\hline$\pi_{m}$ & Recruitment rate into susceptible male population & 0.3 & [29] \\
\hline$\pi_{f}$ & Recruitment rate into susceptible female population & 0.45 & [29] \\
\hline$\alpha_{f}$ & Transmission probability of female with syphilis infection & 0.2 & [15] \\
\hline$\alpha_{m}$ & Transmission probability of male with syphilis infection & 0.5 & [15] \\
\hline$\gamma_{m}$ & $\begin{array}{l}\text { Progression rate from male with primary syphilis to male } \\
\text { with secondary syphilis infection }\end{array}$ & 0.01 & [15] \\
\hline$\gamma_{f}$ & Progression rate from female with primary syphilis & 0.627 & [15] \\
\hline$\psi$ & $\begin{array}{l}\text { Average number of sexual partner per unit time to } \\
\text { female with secondary syphilis infection }\end{array}$ & 2 & [19] \\
\hline$\beta_{m}$ & $\begin{array}{l}\text { Progression rate from male with secondary syphilis to } \\
\text { male with latent syphilis infection }\end{array}$ & 0.618 & [15] \\
\hline$\beta_{f}$ & $\begin{array}{l}\text { Progression rate from female with secondary syphilis to } \\
\text { female individual with latent syphilis infection }\end{array}$ & 0.618 & {$[15]$} \\
\hline$\varphi_{m}$ & Rate of recovery from syphilis in infectious male & 0.1 & [29] \\
\hline$\varphi_{f}$ & Rate of recovery from syphilis in infectious female & 0.1 & [29] \\
\hline$\sigma_{m_{1}}$ & Treatment rate of male with primary stage syphilis & 0.05 & [29] \\
\hline$\sigma_{m_{2}}$ & Treatment rate of male with secondary stage syphilis & 0.1 & [29] \\
\hline$\sigma_{m_{3}}$ & Treatment rate of male with latent stage syphilis & 0.2 & [29] \\
\hline$\rho_{f_{1}}$ & Treatment rate of female with primary stage syphilis & 0.05 & [29] \\
\hline$\rho_{f_{2}}$ & Treatment rate of female with secondary stage syphilis & 0.1 & [29] \\
\hline$\rho_{f_{3}}$ & Treatment rate of female with latent stage syphilis & 0.2 & [29] \\
\hline$\mu$ & Rate of natural death & $5.48 \times 10^{-5}$ day $^{-1}$ & [42] \\
\hline$A_{1}$ & $\begin{array}{l}\text { Weight coefficients for both infectious male and female } \\
\text { syphilis }\end{array}$ & 31 & [38] \\
\hline$B_{1}$ & $\begin{array}{l}\text { Relative cost for prevention and treatment in male and } \\
\text { female individuals }\end{array}$ & 0.5 & [38] \\
\hline$B_{2}$ & $\begin{array}{l}\text { Relative cost for prevention and treatment in male and } \\
\text { female individuals }\end{array}$ & 0.4 & Assumed \\
\hline$B_{3}$ & $\begin{array}{l}\text { Relative cost for prevention and treatment in male and } \\
\text { female individuals }\end{array}$ & 0.3 & Assumed \\
\hline
\end{tabular}

\subsection{Basic properties}

Theorem 1 Let $S_{m}(0), I_{m p}(0), I_{m s}(0), L_{m}(0), R_{m}(0), S_{f}(0), I_{f p}(0), I_{f s}(0), L_{f}(0), R_{f}(0)>0$ be nonnegative initial conditions. Then system (2) has a nonnegative solution $S_{m}(t), I_{m p}(t), I_{m s}(t)$, $L_{m}(t), R_{m}(t), S_{f}(t), I_{f p}(t), I_{f_{s}}(t), L_{f}(t), R_{f}(t)>0$ for all $t>0$. Moreover, $\lim _{\sup _{t \rightarrow \infty}} N(t) \leq$ $\frac{\pi_{m}+\pi_{f}}{\mu}$. In addition, if $N(0) \leq \frac{\pi_{m}+\pi_{f}}{\mu}$, then $N(t) \leq \frac{\pi_{m}+\pi_{f}}{\mu}$ is the feasible region for system (2)

$$
\Omega=\left\{\left(S_{m}, I_{m p}, I_{m s}, L_{m}, R_{m}, S_{m}, I_{f p}, I_{f s}, L_{f}, R_{f}\right) \in \mathbb{R}_{+}^{10}: N_{P} \leq \frac{\pi_{m}+\pi_{f}}{\mu}\right\}
$$

is positively invariant and attracting with respect to system (2). 
Proof From the first equation of system (2) we have

$$
\frac{d S_{m}}{d t}+\frac{\left.\alpha_{f} \psi\left(I_{f p}(t)+I_{f s}(t)\right)+L_{f}(t)\right)}{N(t)} S_{m}(t)+\mu S_{m}(t) \geq 0
$$

From time $t=0$ to $t=t$, integrating (6), we get

$$
\frac{d}{d t}\left[S_{m}(t) \exp \left\{\int_{0}^{t} \frac{\alpha_{f} \psi\left(I_{f p}(t)+I_{f s}(t)+L_{f}(t)\right)}{N(t)}(\bar{\omega}) d \bar{\omega}+\mu t\right\}\right] \geq 0
$$

This means that

$$
S_{m}(t) \geq S_{m}(0) \exp \left\{-\left(\int_{0}^{t} \frac{\alpha_{f} \psi\left(I_{f p}(t)+I_{f s}(t)+L_{f}(t)\right)}{N(t)}(\bar{\omega}) d \bar{\omega}+\mu t\right)\right\}>0, \quad \forall t>0 .
$$

We applied a similar method to establish that $I_{m p}(t), I_{m s}(t), L_{m}(t), R_{m}(t), S_{f}(t), I_{f p}(t), I_{f s}(t)$, $L_{f}(t), R_{f}(t)>0$ remain nonnegative for all $t>0$. Equation (4) is used to prove the second part of the theorem, which says that model system (2) is positively invariant, so that $N(t) \leq \frac{\left(\pi_{m}+\pi_{f}\right)\left(1-\exp ^{-\mu t}\right)}{\mu}+\frac{\mu N(0) \exp ^{-\mu t}}{\mu}$. It follows that as $t \longrightarrow \infty N(t) \leq \frac{\pi_{m}+\pi_{f}}{\mu}$. Furthermore, if $N(0) \leq \frac{\pi_{m}+\pi_{f}}{\mu}$, then $N(t) \leq \frac{\pi_{m}+\pi_{f}}{\mu}$. This establishes that $\Omega$ is the manifold on which the population has nonzero size.

This proves the boundedness of the solutions inside $\Omega$. Hence the solutions to system (2) are positively invariant and attracting in a region $\Omega$. Note that system (2) is feasible biologically and mathematically well posed in $\Omega$ from Theorem 1 .

\section{Analysis of the syphilis model}

This section establishes the syphilis-free equilibrium state, syphilis-present equilibrium state, derived the basic reproduction number, and carryout stability analysis.

\subsection{Equilibrium points}

The equilibrium points of system (2) are established by setting it to zero:

$$
\left\{\begin{array}{l}
\frac{d S_{m}}{d t}=\pi_{m}+\varphi_{m} R_{m}-\alpha_{f} \psi\left(\frac{I_{f p}+I_{f s}+L_{f}}{N}\right) S_{m}-\mu S_{m}=0, \\
\frac{d I_{m p}}{d t}=\alpha_{f} \psi\left(\frac{I_{f p}+I_{f_{s}}+L_{f}}{N}\right) S_{m}-\gamma_{m} I_{m p}-\mu I_{m p}-\sigma_{m 1} I_{m p}=0, \\
\frac{d I_{m s}}{d t}=\gamma_{m} I_{m} p-\beta_{m} I_{m s}-\sigma_{m_{2}} I_{m s}-\mu I_{m s}=0, \\
\frac{d L_{m}}{d t}=\beta_{m} I_{m s}-\mu L_{m}-\sigma_{m_{3}} L_{m}=0, \\
\frac{d R_{m}}{d t}=\sigma_{m 1} I_{m p}+\sigma_{m_{2}} I_{m s}+\sigma_{m 3} L_{m}-\mu R_{m}-\varphi_{m} R_{m}=0, \\
\frac{d S_{f}}{d t}=\pi_{f}+\varphi_{f} R_{f}-\alpha_{m} \psi\left(\frac{I_{m p}+I_{m s}+L_{m}}{N}\right) S_{f}-\mu S_{f}=0, \\
\frac{d I_{f p}}{d t}=\alpha_{m} \psi\left(\frac{I_{m p}+I_{m s}+L_{m}}{N}\right) S_{f}-\gamma_{f} I_{f} p-\mu I_{f} p-\rho_{f_{1}} I_{f p}=0, \\
\frac{d I_{f s}}{d t}=\gamma_{f} I_{f} p-\beta_{f} I_{f s}-\rho_{f_{2}} I_{f s}-\mu I_{f s}=0, \\
\frac{d L_{f}}{d t}=\beta_{f} I_{f s}-\mu L_{f}-\rho_{m_{3}} L_{f}, \\
\frac{d R_{f}}{d t}=\rho_{f_{1}} I_{f p}+\rho_{f_{2}} I_{f s}+\rho_{f_{3}} L_{f}-\mu R_{f}-\varphi_{f} R_{f}=0 .
\end{array}\right.
$$

When there is no syphilis infection, system (2) has a steady state, which is termed a syphilis-free equilibrium. To obtain the nature of stability of the syphilis-free equilibrium, 
we computed and evaluated the Jacobian of system (2) at syphilis-free equilibrium. The local stability of the syphilis-free equilibrium is determined using the signs of the eigenvalues of the Jacobian. The syphilis-free equilibrium for system (2) is

$$
\left(S_{m}^{0}, I_{m p}^{0}, I_{m s}^{0}, L_{m}^{0}, R_{m}^{0}, S_{f}^{0}, I_{f p}^{0}, I_{f s}^{0}, L_{f}^{0}, R_{f}^{0}\right)=\left(\frac{\pi_{m}}{\mu}, 0,0,0,0, \frac{\pi_{f}}{\mu}, 0,0,0,0\right)
$$

This simply means that in the absence of syphilis infection the susceptible males and females proportionally change with their recruitment rate to their death rate.

For the second equilibrium point, let $E^{* *}=\left(S_{m}^{* *}, I_{m p}^{* *}, I_{m s}^{* *}, L_{m}^{* *}, R_{m}^{* *}, S_{f}^{* *}, I_{f p}^{* *}, I_{f s}^{* *}, L_{f}^{* *}, R_{f}^{* *}\right)$ be the syphilis-present equilibrium of model (2). At equilibrium state, let $\lambda_{m}^{* *}=$ $\frac{\alpha_{m} \psi\left(I_{m p}^{* *}+I_{m s}^{* *}+L_{m}^{* *}\right)}{N^{* *}}, \lambda_{f}^{* *}=\frac{\alpha_{f} \psi\left(I_{f p}^{* *}+I_{f s}^{* *}+L_{f}^{* *}\right)}{N^{* *}}$ be the forces of infection, and let $N^{* *}=S_{m}^{* *}+I_{m p}^{* *}+$ $I_{m s}^{* *}+L_{m}^{* *}+R_{m}^{* *}+S_{f}^{* *}+I_{f p}^{* *}+I_{f s}^{* *}+L_{f}^{* *}+R_{f}^{* *}$. Then solving system (2) at steady state yields

$$
\left\{\begin{array}{l}
S_{m}^{* *}=\frac{k_{1} k_{2} I_{m s}^{*}}{\gamma_{m}\left(\lambda_{f}^{* *}+\mu\right)}, \\
I_{m p}^{* *}=\frac{k_{2} I_{m s}^{*}}{\gamma_{m}}, \\
I_{m s}^{* *}=\frac{\pi_{m} \gamma_{m} k_{3} k_{4}}{k_{1} k_{2} k_{3} k_{4}-\left(\sigma_{m_{3}} \beta_{m} \gamma_{m} \varphi_{m}+k_{3} \sigma_{m_{2}} \gamma_{m} \psi_{m}+\varphi_{m} \sigma_{m_{1}} k_{2} k_{3}\right)} \\
L_{m}^{* *}=\frac{\beta_{m} I_{m s}^{*}}{k_{3}}, \\
R_{m}^{* *}=\frac{k_{1} k_{2} I_{m s}^{* *}-\gamma_{m} \pi_{m}}{\gamma_{m} \varphi_{m}}, \\
S_{f}^{* *}=\frac{h_{1} h_{2} I_{f s}^{* *}}{\gamma_{f}\left(\lambda_{m}^{*}+\mu\right)}, \\
I_{f p}^{* *}=\frac{h_{2} I_{f s}^{*}}{\gamma_{f}}, \quad \pi_{f} \gamma_{f} h_{3} h_{4} \\
I_{f s}^{* *}=\frac{{ }_{1} h_{1} h_{2} h_{3} h_{4}-\left(\rho_{f_{3}} \beta_{f} \gamma_{f} \varphi_{f}+h_{3} \rho_{f_{2}} \gamma_{f} \psi_{f}+\varphi_{f} \rho_{f_{1}} h_{2} h_{3}\right)}{\left.h_{1}\right)} \\
L_{f}^{* *}=\frac{\beta_{f} I_{f s}^{* *}}{h_{3}}, \\
R_{f}^{* *}=\frac{\left.\left(\gamma_{f}+\mu+\rho_{f_{1}}\right)\left(\beta_{f}+\rho_{f_{2}}+\mu\right)\right)_{f s}^{* *}-\gamma_{f} \pi_{f}}{\gamma_{f} \varphi_{2}}
\end{array}\right.
$$

where $k_{1}=\gamma_{m}+\mu+\sigma_{m_{1}}, k_{2}=\beta_{m}+\sigma_{m_{2}}+\mu, k_{3}=\mu+\sigma_{m_{3}}, k_{4}=\mu+\varphi_{m}$, and $h_{1}=\gamma_{f}+\mu+\rho_{f_{1}}$, $h_{2}=\beta_{f}+\rho_{f_{2}}+\mu, h_{3}=\mu+\rho_{f_{3}}, h_{4}=\mu+\varphi_{f}$.

\subsection{Basic reproduction number}

The average number of secondary infections caused by one infectious person when the entire population is susceptible is termed the basic reproduction number $\mathcal{R}_{0}$. The epidemiological threshold of syphilis is denoted by $\mathcal{R}_{0}=\rho\left(F V^{-1}\right)$, where $\rho$ is the dominant eigenvalue. To find the basic reproduction number of system (2), we adopted the techniques in [41] to get

$$
F=\left(\begin{array}{c}
\alpha_{f} \psi\left(\frac{\left(I_{f p}+I_{f_{s}}+L_{f}\right.}{N}\right) S_{m} \\
0 \\
0 \\
\alpha_{m} \psi\left(\frac{I_{m p}+I_{m s}+L_{m}}{N}\right) S_{f} \\
0 \\
0
\end{array}\right) \quad \text { and } \quad V=\left(\begin{array}{c}
\left(\gamma_{m}+\mu+\sigma_{m_{1}}\right) I_{m p} \\
-\gamma_{m} I_{m p}+\left(\beta_{m}+\mu+\sigma_{m_{2}}\right) I_{m s} \\
-\beta_{m} I_{m s}+\left(\mu+\sigma_{m_{3}}\right) L_{m} \\
\left(\gamma_{f}+\mu+\rho_{f_{1}}\right) I_{f p} \\
-\gamma_{f} I_{f p}+\left(\beta_{f}+\mu+\rho_{f_{2}}\right) I_{f_{s}} \\
-\beta_{f} I_{f s}+\left(\mu+\rho_{f_{3}}\right) L_{f}
\end{array}\right) \text {. }
$$


The matrices $F$ and $V$ contain new infection terms and transition terms, respectively, in system (2). Evaluating the Jacobian matrices of $F$ and $V$ at syphilis-free equilibrium yields

$$
F=\left(\begin{array}{cccccc}
0 & 0 & 0 & \frac{\alpha_{f} \psi \pi_{m}}{\pi_{m}+\pi_{f}} & \frac{\alpha_{f} \psi \pi_{m}}{\pi_{m}+\pi_{f}} & \frac{\alpha_{f} \psi \pi_{m}}{\pi_{m}+\pi_{f}} \\
0 & 0 & 0 & 0 & 0 & 0 \\
0 & 0 & 0 & 0 & 0 & 0 \\
\frac{\alpha_{m} \psi \pi_{f}}{\pi_{m}+\pi_{f}} & \frac{\alpha_{m} \psi \pi_{f}}{\pi_{m}+\pi_{f}} & \frac{\alpha_{m} \psi \pi_{f}}{\pi_{m}+\pi_{f}} & 0 & 0 & 0 \\
0 & 0 & 0 & 0 & 0 & 0 \\
0 & 0 & 0 & 0 & 0 & 0
\end{array}\right)
$$

and

$$
V=\left(\begin{array}{cccccc}
\gamma_{m}+\mu+\sigma_{m_{1}} & 0 & 0 & 0 & 0 & 0 \\
-\gamma_{m} & \beta_{m}+\mu+\sigma_{m_{2}} & 0 & 0 & 0 & 0 \\
0 & -\beta_{m} & \mu+\sigma_{m_{3}} & 0 & 0 & 0 \\
0 & 0 & 0 & \gamma_{f}+\mu+\rho_{f_{1}} & 0 & 0 \\
0 & 0 & 0 & -\gamma_{f} & \beta_{f}+\mu+\rho_{f_{2}} & 0 \\
0 & 0 & 0 & 0 & -\beta_{f} & \mu+\rho_{f_{3}}
\end{array}\right) .
$$

Therefore system (2) has the basic reproduction number

$$
\mathcal{R}_{0}=\sqrt{\frac{\psi^{2} \alpha_{f} \alpha_{m} \pi_{m} \pi_{f}\left(\beta_{m} \gamma_{m}+\gamma_{m} q_{2}+q_{2} q_{3}\right)\left(\beta_{f} \gamma_{f}+\gamma_{f} q_{6}+q_{5} q_{6}\right)}{\left(\pi_{m}+\pi_{f}\right)^{2} q_{1} q_{2} q_{3} q_{4} q_{5} q_{6}}}
$$

where $q_{1}=\left(\gamma_{m}+\mu+\sigma_{m_{1}}\right), q_{2}=\left(\beta_{m}+\mu+\sigma_{m_{2}}\right) q_{3}=\left(\mu+\sigma_{m_{3}}\right), q_{4}=\left(\gamma_{f}+\mu+\rho_{f_{1}}\right)$, $q_{5}=\left(\beta_{f}+\mu+\rho_{f_{2}}\right)$, and $q_{6}=\left(\mu+\rho_{f_{3}}\right)$.

\subsection{Local stability of the syphilis-free equilibrium}

Theorem 2 The syphilis-free equilibrium of system (2) is locally asymptotically stable if $\mathcal{R}_{0}<1$ and unstable otherwise.

Proof The Jacobian matrix of system (2) at syphilis-free equilibrium is

$$
J\left(E^{0}\right)=\left(\begin{array}{cccccccccc}
-r_{1} & 0 & 0 & 0 & \varphi_{1} & 0 & -\frac{\alpha_{f} \psi \pi_{m}}{\pi_{m}+\pi_{f}} & -\frac{\alpha_{f} \psi \pi_{m}}{\pi_{m}+\pi_{f}} & -\frac{\alpha_{f} \psi \pi_{m}}{\pi_{m}+\pi_{f}} & 0 \\
0 & -r_{2} & 0 & 0 & 0 & 0 & \frac{\alpha_{f} \psi \pi_{m}}{\pi_{m}+\pi_{f}} & \frac{\alpha_{f} \psi \pi_{m}}{\pi_{m}+\pi_{f}} & \frac{\alpha_{f} \psi \pi_{m}}{\pi_{m}+\pi_{f}} & 0 \\
0 & \gamma_{m} & -r_{3} & 0 & 0 & 0 & 0 & 0 & 0 & 0 \\
0 & 0 & \beta_{m} & -r_{4} & 0 & 0 & 0 & 0 & 0 & 0 \\
0 & \sigma_{m_{1}} & \sigma_{m_{2}} & \sigma_{m_{3}} & -r_{5} & 0 & 0 & 0 & 0 & 0 \\
0 & -\frac{\alpha_{f} \psi \pi_{f}}{\pi_{m}+\pi_{f}} & -\frac{\alpha_{f} \psi \pi_{f}}{\pi_{m}+\pi_{f}} & -\frac{\alpha_{f} \psi \pi_{f}}{\pi_{m}+\pi_{f}} & 0 & -r_{6} & 0 & 0 & 0 & \varphi_{f} \\
0 & \frac{\alpha_{m} \psi \pi_{f}}{\pi_{m}+\pi_{f}} & \frac{\alpha_{m} \psi \pi_{f}}{\pi_{m}+\pi_{f}} & \frac{\alpha_{m} \psi \pi_{f}}{\pi_{m}+\pi_{f}} & 0 & 0 & -r_{7} & 0 & 0 & 0 \\
0 & 0 & 0 & 0 & 0 & 0 & \gamma_{f} & -r_{8} & 0 & 0 \\
0 & 0 & 0 & 0 & 0 & 0 & 0 & \beta_{f} & -r_{9} & 0 \\
0 & 0 & 0 & 0 & 0 & 0 & \rho_{f_{1}} & \rho_{f_{2}} & \rho_{f_{3}} & -r_{10}
\end{array}\right),
$$

where $r_{1}=\mu, r_{2}=\left(\gamma_{m}+\mu+\sigma_{m_{1}}\right), r_{3}=\left(\beta_{m}+\mu+\sigma_{m_{2}}\right), r_{4}=\left(\mu+\sigma_{m_{3}}\right), r_{5}=\left(\mu+\varphi_{m}\right), r_{6}=\mu$, $r_{7}=\left(\gamma_{f}+\mu+\rho_{f_{1}}\right), r_{8}=\left(\gamma_{f}+\mu+\rho_{f_{2}}\right), r_{9}=\left(\mu+\rho_{f_{3}}\right)$, and $r_{10}=\left(\mu+\varphi_{f}\right)$. 
From the matrix $J\left(E^{0}\right)$ we have $\lambda_{1}=-\mu<0, \lambda_{2}=-\left(\mu+\varphi_{m}\right)<0, \lambda_{3}=-\mu<0$, and $\lambda_{4}=$ $-\left(\mu+\varphi_{f}\right)<0$, so that the matrix $J\left(E^{0}\right)$ reduces to

$$
\left(\begin{array}{cccccc}
-r_{2} & 0 & 0 & \frac{\alpha_{f} \psi \pi_{m}}{\pi_{m}+\pi_{f}} & \frac{\alpha_{f} \psi \pi_{m}}{\pi_{m}+\pi_{f}} & \frac{\alpha_{f} \psi \pi_{m}}{\pi_{m}+\pi_{f}} \\
\gamma_{m} & -r_{3} & 0 & 0 & 0 & 0 \\
0 & \beta_{m} & -r_{4} & 0 & 0 & 0 \\
\frac{\alpha_{m} \psi \pi_{f}}{\pi_{m}+\pi_{f}} & \frac{\alpha_{m} \psi \pi_{f}}{\pi_{m}+\pi_{f}} & \frac{\alpha_{m} \psi \pi_{f}}{\pi_{m}+\pi_{f}} & -r_{7} & 0 & 0 \\
0 & 0 & 0 & \gamma_{f} & -r_{8} & 0 \\
0 & 0 & 0 & 0 & \beta_{f} & -r_{9}
\end{array}\right) .
$$

The characteristic equation for (11) is hereby defined as follows:

$$
\lambda^{6}+D_{1} \lambda^{5}+D_{2} \lambda^{4}+D_{3} \lambda^{3}+D_{4} \lambda^{2}+D_{5} \lambda+D_{6}=0
$$

where the coefficients of (12) are given as $D_{1}=\left(r_{2}+r_{3}+r_{4}+r_{7}+r_{8}+r_{9}\right), D_{2}=r_{9}\left(r_{2}+r_{3}+r_{4}+\right.$ $\left.r_{7}+r_{8}\right)+r_{3}\left(r_{2}+r_{3}\right)+r_{2} r_{3}+r_{7}\left(r_{2}+r_{3}+r_{4}\right)+r_{8}\left(r_{2}+r_{3}+r_{4}+r_{7}\right)-b_{1} c_{1}, D_{3}=\left(r_{9}\left(r_{4}\left(r_{2}+r_{3}\right)+r_{2} r_{3}+\right.\right.$ $\left.r_{7}\left(r_{2}+r_{3}+r_{4}\right)+r_{8}\left(r_{2}+r_{3}+r_{4}+r_{7}\right)\right)+r_{7}\left(r_{4}\left(r_{2}+r_{3}\right)+r_{2} r_{3}\right)+r_{8}\left(r_{4}\left(r_{2}+r_{3}\right)+r_{2} r_{3}+r_{7}\left(r_{2}+r_{3}+r_{4}\right)\right)+$ $\left.b_{1} c_{1} r_{2}+r_{2} r_{3} r_{4}-b_{1} c_{1}\left(2+\gamma_{f}+\gamma_{m}\right)+\left(r_{2}+r_{3}+r_{4}\right)\right), D_{4}=\left(c_{1}\left(\gamma_{m} b_{1} r_{2}+\gamma_{m} b_{1} r_{3}\right)+r_{8}\left(r_{7}\left(r_{4}\left(r_{2}+\right.\right.\right.\right.$ $\left.\left.\left.r_{3}\right)+r_{2} r_{3}\right)-\gamma_{m} b_{1} c_{1}+b_{1} c_{1} r_{2}+r_{2} r_{3} r_{4}-b_{1} c_{1}\left(r_{2}+r_{3}+r_{4}\right)\right)-\left(\gamma_{m} b_{1} c_{1}-b_{1} c_{1} r-2\right)\left(r_{2}+r_{3}+r_{4}\right)+$ $\gamma_{f}\left(b_{1} c_{1} r_{2}-\gamma_{m} b_{1} c_{1}+b_{1} c_{1} r_{7}\right)+r_{9}\left(r_{7}\left(r_{4}\left(r_{2}+r_{3}\right)+r_{2} r_{3}\right)+r_{8}\left(r_{4}\left(r_{2}+r_{3}\right)-b_{1} c_{1}+r_{2} r_{3}+r_{7}\left(r_{2}+r_{3}+\right.\right.\right.$ $\left.\left.\left.r_{4}\right)\right)-\gamma_{f} b_{1} c_{1}-\gamma_{m} b_{1} c_{1}+b_{1} c_{1} r_{2}+r_{2} r_{3} r_{4}-b_{1} c_{1}\left(r_{2}+r_{3}+r_{4}\right)\right)-b_{1} c_{1} r_{2}^{2}-b_{1} c_{1}\left(r_{4}\left(r_{2}+r_{3}\right)+r_{2} r_{3}\right)+$ $\left.r_{2} r_{3} r_{4} r_{7}-\gamma_{f} b_{1} c_{1}\left(r_{2}+r_{3}+r_{4}+r_{7}\right)-\beta_{f} \gamma_{f} b_{1} c_{1}-\beta_{m} \gamma_{m} b_{1} c_{1}\right), D_{5}=\left(\beta_{f}\left(\gamma_{f}\left(b_{1} c_{1} r_{2}-\gamma_{m} b_{1} c_{1}+\right.\right.\right.$ $\left.\left.b_{1} c_{1} r_{7}\right)+\gamma_{f} b_{1} c_{1} r_{8}\right)-\gamma_{f}\left(r_{7}\left(b_{1} c_{1} r_{2}-\gamma_{m} b_{1} c_{1}+b_{1} c_{1} r_{7}\right)-c_{1}\left(\gamma_{m} b_{1} r_{2}+\gamma_{m} b_{1} r_{3}\right)+c_{1}\left(c_{1} b_{1}^{2}+b_{1} r_{2}^{2}\right)+\right.$ $\left.\beta_{m} \gamma_{m} b_{1} c-1\right)-r_{9}\left(\left(\gamma_{m} b_{1} c_{1}-b_{1} c_{1} r_{2}\right)\left(r_{2}+r_{3}+r_{4}\right)-r_{8}\left(r_{7}\left(r_{4}\left(r_{2}+r_{3}\right)+r_{2} r_{3}\right)-\gamma_{m} b_{1} c_{1}+b_{1} c_{1} r_{2}+\right.\right.$ $\left.r_{2} r_{3} r_{4}-b_{1} c_{1}\left(r_{2}+r_{3}+r_{4}\right)\right)-c_{1}\left(\gamma_{m} b_{1} r_{2}+\gamma_{m} b_{1} r_{3}\right)-\gamma_{f}\left(b_{1} c_{1} r_{2}-\gamma_{m} b_{1} c_{1}+b_{1} c_{1} r_{7}\right)+b_{1} c_{1} r_{2}^{2}+$ $\left.b_{1} c_{1}\left(r_{4}\left(r_{2}+r_{3}\right)+r_{2} r-3\right)-r_{2} r_{3} r_{4} r_{7}+\gamma_{f} b_{1} c-1\left(r_{2}+r_{3}+r_{4}+r_{7}\right)+\beta_{m} \gamma_{m} b_{1} c-1\right)-r_{8}\left(\left(\gamma_{m} b_{1} c_{1}-\right.\right.$ $\left.b_{1} c_{1} r_{2}\right)\left(r_{2}+r_{3}+r_{4}\right)-c_{1}\left(\gamma_{m} b_{1} r_{2}+\gamma_{m} b_{1} r_{3}\right)+b_{1} c_{1} r_{2}^{2}+b_{1} c_{1}\left(r_{4}\left(r_{2}+r_{3}\right)+r_{2} r_{3}\right)-r_{2} r-3 r-4 r_{7}+$ $\left.\beta_{m} \gamma_{m} b_{1} c_{1}\right)+\gamma_{f}\left(b_{1} c_{1} r_{2}-\gamma_{m} b_{1} c_{1}+b_{1} c_{1} r_{7}\right)\left(r_{2}+r_{3}+r_{4}+r_{7}\right)-\gamma_{f} b_{1} c_{1}\left(r_{4}\left(r_{2}+r_{3}\right)-b_{1} c_{1}+r_{2} r_{3}+\right.$ $\left.\left.r_{7}\left(r_{2}+r_{3}+r_{4}\right)\right)-\beta_{f} \gamma_{f} b_{1} c_{1}\left(r_{2}+r_{3}+r_{4}+r_{7}+r_{8}\right)\right), D_{6}=\left(\beta_{f}\left(\gamma_{f}\left(b_{1} c_{1} r_{2}-\gamma_{m} b_{1} c_{1}+b_{1} c_{1} r_{7}\right)+\right.\right.$ $\left.\gamma_{f} b_{1} c_{1} r_{8}\right)\left(r_{2}+r_{3}+r_{4}+r_{7}+r_{8}\right)-r_{9}\left(\gamma_{f}\left(r_{7}\left(b_{1} c_{1} r_{2}-\gamma_{m} b_{1} c_{1}+b_{1} c_{1} r_{7}\right)-c_{1}\left(\gamma_{m} b_{1} r_{2}+\gamma_{m} b_{1} r_{3}\right)+\right.\right.$ $\left.c_{1}\left(c_{1} b_{1}^{2}+b_{1} r_{2}^{2}\right)+\beta_{m} \gamma_{m} b_{1} c_{1}\right)+r_{8}\left(\left(\gamma_{m} b_{1} c_{1}-b_{1} c_{1} r_{2}\right)\left(r_{2}+r_{3}+r_{4}\right)-c_{1}\left(\gamma_{m} b_{1} r_{2}+\gamma_{m} b_{1} r_{3}\right)+b_{1} c_{1} r_{2}^{2}+\right.$ $\left.b_{1} c_{1}\left(r_{4}\left(r_{2}+r_{3}\right)+r_{2} r_{3}\right)-r_{2} r_{3} r_{4} r_{7}+\beta_{m} \gamma_{m} b_{1} c_{1}\right)-\gamma_{f}\left(b_{1} c_{1} r_{2}-\gamma_{m} b_{1} c_{1}+b_{1} c_{1} r_{7}\right)\left(r_{2}+r_{3}+r_{4}+\right.$ $\left.\left.r_{7}\right)+\gamma_{f} b_{1} c_{1}\left(r_{4}\left(r_{2}+r_{3}\right)-b_{1} c_{1}+r_{2} r_{3}+r_{4}\left(r_{2}+r_{3}+r_{4}\right)\right)\right)-\beta_{f}\left(\gamma_{f}\left(r_{7}\left(b_{1} c_{1} r_{2}-\gamma_{m} b_{1} c_{1}+b_{1} c_{1} r_{7}\right)-\right.\right.$ $\left.c_{1}\left(\gamma_{m} b_{1} r_{2}+\gamma_{m} b_{1} r_{1}\right)+c_{1}\left(c_{1} b_{1}^{2}+b_{1} r_{2}^{2}\right)+\beta_{m} \gamma_{m} b_{1} c-1\right)+r_{8}\left(\gamma_{f}\left(b_{1} c_{1} r_{2}-\gamma_{m} b_{1} c_{1}+b_{1} c_{1} r_{7}\right)+\right.$ $\left.\left.\left.\gamma_{f} b_{1} c_{1} r_{8}\right)\right)-\beta_{f} \gamma_{f} b_{1} c_{1}\left(r_{4}\left(r_{2}+r_{3}\right)-b_{1} c_{1}+r_{2} r_{3}+r_{7}\left(r_{2}+r_{3}+r_{4}\right)+r_{8}\left(r_{2}+r_{3}+r_{4}+r_{7}\right)\right)\right)$.

By applying the Routh-Hurwitz criterion (which states that all roots of the polynomial equation (12) have a negative real part if and only if the coefficients are positive and the determinant of the matrices $H_{i}>1$ for $\left.i=1, \ldots, 6\right)$ it is clear that $D_{1}>0$. Therefore, if $D_{j}>0$ for $j=2, \ldots, 6$ and the necessary condition for the Routh-Hurwitz criterion for the sixthorder characteristic polynomial in (12) is satisfied, then we conclude that the syphilis-free equilibrium is locally asymptotically stable (LAS).

\subsection{Syphilis global asymptotic stability}

The approach in [8] is adopted to investigate the global asymptotic stability (GAS) of the syphilis-free equilibrium for the model (2). 
Lemma 1 Let system (2) be of the form

$$
\left\{\begin{array}{l}
\frac{d Q}{d t}=F(Q, Z), \\
\frac{d Z}{d t}=G(Q, Z), \quad(Q, 0)=0
\end{array}\right.
$$

where $Q=\left(S_{m}, R_{m}, S_{f}, R_{f}\right)$ and $Z=\left(I_{m p}, I_{m s}, L_{m}, I_{f p}, I_{f s}, L_{m}\right)$, and the components of $Q \in \mathbb{R}^{4}$ represent the population that is not infected, and the components of $Z \in \mathbb{R}^{6}$ represent the infected population [8]. Consider the syphilis-free equilibrium $E^{0}=\left(Q^{0}, 0\right)$, where

$$
Q^{0}=\left(\frac{\pi_{m}}{\mu}, 0,0,0,0, \frac{\pi_{f}}{\mu}, 0,0,0,0\right) .
$$

The conditions that must be met to guarantee the global asymptotic stability are: $H_{1}: \frac{d Q}{d t}=$ $F\left(Q^{0}, 0\right)$, where $Q^{0}$ is $(G A S) . H_{2}: G(Q, Z)=P Z-\hat{G}(Q, Z), \hat{G}(Q, Z) \geq 0$ for $(Q, Z) \in \Omega$, where $P=D_{z} G\left(Q^{0}, 0\right)$ is an M-matrix, and $\Omega$ is the biological feasible region. Hence $E^{0}$ is $(G A S)$ if $\mathcal{R}_{0}<1$.

Theorem 3 The syphilis-free equilibrium of system (2) is (GAS) if $\mathcal{R}_{0}<1$ and unstable otherwise.

Proof We have to establish that conditions $\left(H_{1}\right)$ and $\left(H_{2}\right)$ hold when $\mathcal{R}_{0}<1$. For the uninfected population, we have

$$
F(Q, 0)=\left(\begin{array}{c}
\pi_{m}-\mu S_{m} \\
0 \\
\pi_{f}-\mu S_{f} \\
0
\end{array}\right)
$$

Denoting by $Q \in \mathbb{R}^{6}$ the infected compartments in model (2), we have $G(Q, Z)=P Z$ $\hat{G}(Q, Z)$, where

$$
P=\left(\begin{array}{cccccc}
-\left(\gamma_{m}+\mu+\sigma_{m_{1}}\right) & 0 & 0 & \frac{\alpha_{f} S_{m}}{N} & \frac{\alpha_{f} S_{m}}{N} & \frac{\alpha_{f} S_{m}}{N} \\
\gamma_{m} & -\left(\beta_{m}+\mu+\sigma_{m_{2}}\right) & 0 & 0 & 0 & 0 \\
0 & \beta_{1} S_{f} & -\left(\mu+\sigma_{m_{3}}\right) & 0 & 0 & 0 \\
\frac{\alpha_{m} S_{f}}{N} & \frac{\alpha_{m} S_{f}}{N} & \frac{\alpha_{m} S_{f}}{N} & -\left(\gamma_{f}+\mu+\rho_{f_{1}}\right) & 0 & 0 \\
0 & 0 & 0 & \gamma_{f} & -\left(\beta_{f}+\mu+\rho_{f_{2}}\right) & 0 \\
0 & 0 & 0 & 0 & \beta_{f} & -\left(\mu+\rho_{f_{3}}\right)
\end{array}\right) .
$$

Thus

$$
\hat{G}(Q, Z)=\left(\begin{array}{c}
\hat{G}_{1}(Q, Z) \\
\hat{G}_{2}(Q, Z) \\
\hat{G}_{3}(Q, Z) \\
\hat{G}_{4}(Q, Z) \\
\hat{G}_{5}(Q, Z) \\
\hat{G}_{6}(Q, Z)
\end{array}\right)\left(\begin{array}{c}
\alpha_{1}\left(I_{f p}+I_{f s}+L_{f}\right)\left(1-\frac{S_{m}}{N}\right) \\
0 \\
0 \\
\alpha_{2}\left(I_{m p}+I_{m s}+L_{m}\right)\left(1-\frac{S_{f}}{N}\right) \\
0 \\
0
\end{array}\right)\left(\begin{array}{c}
I_{m p} \\
I_{m s} \\
L_{m} \\
I_{f p} \\
I_{f s} \\
L_{f}
\end{array}\right) .
$$

Since $S_{m}<N$ and $S_{f}<N$, we have $\hat{G}_{1}(Q, Z), \hat{G}_{2}(Q, Z), \hat{G}_{3}(Q, Z), \hat{G}_{4}(Q, Z), \hat{G}_{5}(Q, Z)$, $\hat{G}_{6}(Q, Z) \geq 0$. The global stability of $Q^{0}=\left(\frac{\pi_{m}}{\mu}, 0,0,0,0, \frac{\pi_{f}}{\mu}, 0,0,0,0\right)$ of the system $\frac{d Q}{d t}=$ 
$F\left(Q^{0}, 0\right)$ is easy to verify. Therefore $Q^{0}$ is globally asymptotically stable if $\mathcal{R}_{0}<1$. This completes the proof.

\subsection{Bifurcation analysis of the syphilis model}

In this section, we adopt the techniques established in $[7,9,16]$ to study the bifurcation analysis for the syphilis system (2). We apply center manifold theory [24] to the syphilis system (2) by taking $\mathcal{R}_{0}=1$ if and only if

$$
\alpha_{f}=\alpha_{f}^{*}=\frac{\left(q_{1} q_{2} q_{3} q_{4} q_{5} q_{6}\left(\pi_{m}+\pi_{f}\right)\right)}{\psi^{2} \alpha_{m} \pi_{m} \pi_{f}\left(\beta_{m} \gamma_{m}+\gamma_{m} q_{2}+q_{2} q_{3}\right)\left(\beta_{f} \gamma_{2}+\gamma_{2} q_{6}+q_{5} q_{6}\right)} .
$$

We introduce a new set of variables for the syphilis model (2) for convenience sake by letting $x_{1}=S_{m}, x_{2}=I_{m p}, x_{3}=I_{m s}, x_{4}=L_{m}, x_{5}=R_{m}, x_{6}=S_{f}, x_{7}=I_{f p}, x_{8}=I_{f s}, x_{9}=L_{f}, x_{10}=R_{f}$, $x=\left(x_{1}, x_{2}, x_{3}, x_{4}, x_{5}, x_{6}, x_{7}, x_{8}, x_{9}, x_{10}\right)^{T}$, and $f=\left(f_{1}, f_{2}, f_{3}, f_{4}, f_{5}, f_{6}, f_{7}, f_{8}, f_{9}, f_{10}\right)^{T}$. Thus we write model (2) in the form of the differential equation

$$
\frac{d x}{d t}=f\left(x, \alpha_{f}\right)
$$

that is,

$$
\left\{\begin{array}{l}
f_{1}=\pi_{m}+\varphi_{m} x_{5}-\alpha_{f} \psi\left(\frac{x_{7}+x_{8}+x_{9}}{x_{1}+x_{2}+x_{3}+x_{4}+x_{5}+x_{6}+x_{7}+x_{8}+x_{9}+x_{10}}\right) x_{1}-\mu x_{1}, \\
f_{2}=\alpha_{f} \psi\left(\frac{x_{7}+x_{8}+x_{9}}{x_{1}+x_{2}+x_{3}+x_{4}+x_{5}+x_{6}+x_{7}+x_{8}+x_{9}+x_{10}}\right) x_{1}-\gamma_{m} x_{2}-\mu x_{2}-\sigma_{m_{1}} x_{2}, \\
f_{3}=\gamma_{m} x_{2}-\beta_{m} x_{3}-\sigma_{m_{2}} x_{3}-\mu x_{3} \\
f_{4}=\beta_{m} x_{3}-\mu x_{4}-\sigma_{m_{3}} x_{4} \\
f_{5}=\sigma_{m_{1}} x_{2}+\sigma_{m_{2}} x_{3}+\sigma_{m_{3}} x_{4}-\mu x_{5}-\varphi_{m} x_{5} \\
f_{6}=\pi_{f}+\varphi_{f} x_{6}-\alpha_{m} \psi\left(\frac{x_{2}+x_{3}+x_{4}}{x_{1}+x_{2}+x_{3}+x_{4}+x_{5}+x_{6}+x_{7}+x_{8}+x_{9}+x_{10}}\right) x_{6}-\mu x_{6}, \\
f_{7}=\alpha_{m} \psi\left(\frac{x_{2}+x_{3}+x_{4}+x_{2}+x_{3}+x_{4}+x_{5}+x_{6}+x_{7}+x_{8}+x_{9}+x_{10}}{x_{1}}\right) x_{6}-\gamma_{f} x_{7}-\mu x_{7}-\rho_{f_{1}} x_{7}, \\
f_{8}=\gamma_{f} x_{7}-\beta_{f} x_{8}-\rho_{f_{2}} x_{8}-\mu x_{8} \\
f_{9}=\beta_{f} x_{8}-\mu x_{9}-\rho_{m_{3}} x_{9}, \\
f_{10}=\rho_{f_{1}} x_{7}+\rho_{f_{2}} x_{8}+\rho_{f_{3}} x_{9}-\mu x_{10}-\varphi_{f} x_{10} .
\end{array}\right.
$$

Computing the Jacobian matrix $J\left(E^{0}, \alpha_{f}\right)$ associated with (17) at syphilis-free equilibrium $E^{0}$ yields

$$
\left(\begin{array}{cccccccccc}
-r_{1} & 0 & 0 & 0 & \varphi_{1} & 0 & -\frac{\alpha_{f} \psi \pi_{m}}{\pi_{m}+\pi_{f}} & -\frac{\alpha_{f} \psi \pi_{m}}{\pi_{m}+\pi_{f}} & -\frac{\alpha_{f} \psi \pi_{m}}{\pi_{m}+\pi_{f}} & 0 \\
0 & -r_{2} & 0 & 0 & 0 & 0 & \frac{\alpha_{f} \psi \pi_{m}}{\pi_{m}+\pi_{f}} & \frac{\alpha_{f} \psi \pi_{m}}{\pi_{m}+\pi_{f}} & \frac{\alpha_{f} \psi \pi_{m}}{\pi_{m}+\pi_{f}} & 0 \\
0 & \gamma_{m} & -r_{3} & 0 & 0 & 0 & 0 & 0 & 0 & 0 \\
0 & 0 & \beta_{m} & -r_{4} & 0 & 0 & 0 & 0 & 0 & 0 \\
0 & \sigma_{m_{1}} & \sigma_{m_{2}} & \sigma_{m_{3}} & -r_{5} & 0 & 0 & 0 & 0 & 0 \\
0 & -\frac{\alpha_{f} \psi \pi_{f}}{\pi_{m}+\pi_{f}} & -\frac{\alpha_{f} \psi \pi_{f}}{\pi_{m}+\pi_{f}} & -\frac{\alpha_{f} \psi \pi_{f}}{\pi_{m}+\pi_{f}} & 0 & -r_{6} & 0 & 0 & 0 & \varphi_{f} \\
0 & \frac{\alpha_{m} \psi \pi_{f}}{\pi_{m}+\pi_{f}} & \frac{\alpha_{m} \psi \pi_{f}}{\pi_{m}+\pi_{f}} & \frac{\alpha_{m} \psi \pi_{f}}{\pi_{m}+\pi_{f}} & 0 & 0 & -r_{7} & 0 & 0 & 0 \\
0 & 0 & 0 & 0 & 0 & 0 & \gamma_{f} & -r_{8} & 0 & 0 \\
0 & 0 & 0 & 0 & 0 & 0 & 0 & \beta_{f} & -r_{9} & 0 \\
0 & 0 & 0 & 0 & 0 & 0 & \rho_{f_{1}} & \rho_{f_{2}} & \rho_{f_{3}} & -r_{10}
\end{array}\right) .
$$


Considering the matrix $J\left(E^{0}, \alpha_{f}\right)$, there exists a simple eigenvalue, and the remaining eigenvalues have negative real parts. As a result, it is possible to apply center and manifold theory to the syphilis model (17). We further compute the right and left eigenvectors of the matrix to get $w_{1}=\frac{\psi_{m} w_{5}}{r_{1}}-\frac{\alpha_{f} \psi \pi_{m}}{\left(\pi_{m}+\pi_{f}\right) r_{1}} w_{7}-\frac{\alpha_{f} \psi \pi_{m}}{\left(\pi_{m}+\pi_{f}\right) r_{1}} w_{8}-\frac{\alpha_{f} \psi \pi_{m}}{\left(\pi_{m}+\pi_{f}\right) r_{1}} w_{9}, w_{3}=\frac{r_{1}}{r_{3}} w_{2}, w_{4}=\frac{\beta_{m} \gamma_{m}^{2}}{r_{4} r_{3}^{2}} w_{2}$, $w_{5}=\left(\frac{\sigma_{m_{1}}}{r_{5}}+\frac{\sigma_{m_{2}} \gamma_{m}}{r_{3} r_{5}}+\frac{\sigma_{m_{3}} \beta_{m} \gamma_{m}}{r_{3} r_{4} r_{5}}\right) w_{2}, w_{6}=-\left(\frac{\alpha_{m} \psi \pi_{m}}{\left(\pi_{m}+\pi_{f}\right) r_{6}}+\frac{\alpha_{m} \psi \pi_{m} \gamma_{m}}{\left(\pi_{m}+\pi_{f}\right) r_{3} r_{6}}+\frac{\alpha_{m} \psi \pi_{m} \beta_{m} \gamma_{m}}{\left(\pi_{m}+\pi_{f}\right) r_{3} r_{4} r_{6}}\right) w_{2}+\frac{\varphi_{m}}{r_{6}} w_{10}$, $w_{7}=\left(\frac{\alpha_{m} \psi \pi_{m}}{\left(\pi_{m}+\pi_{f}\right) r_{7}}+\frac{\alpha_{m} \psi \pi_{m} \gamma_{m}}{\left(\pi_{m}+\pi_{f}\right) r_{3} r_{7}}+\frac{\alpha_{m} \psi \pi_{m} \beta m r_{m}}{\left(\pi_{m}+\pi_{f}\right) r_{3} r_{4} r_{7}}\right) w_{2}, w_{8}=\left(\frac{\alpha_{m} \psi \pi_{m}}{\left(\pi_{m}+\pi_{f}\right) r_{7}}+\frac{\alpha_{m} \psi \pi_{m} \gamma_{m}}{\left(\pi_{m}+\pi_{f}\right) r_{3} r_{7}}+\frac{\alpha_{m} \psi \pi_{m} \beta_{m} \gamma_{m}}{\left(\pi_{m}+\pi_{f}\right) r_{3} r_{4} r_{7}}\right) \frac{\gamma_{2}}{r_{8}} w_{2}$, $w_{9}=\left(\frac{\alpha_{m} \psi \pi_{m}}{\left(\pi_{m}+\pi_{f}\right) r_{7}}+\frac{\alpha_{m} \psi \pi_{m} \gamma_{m}}{\left(\pi_{m}+\pi_{f}\right) r_{3} r_{7}}+\frac{\alpha_{m} \psi \pi_{m} \beta_{m} \gamma_{m}}{\left(\pi_{m}+\pi_{f}\right) r_{3} r_{4} r_{7}} \frac{\gamma_{2} \beta_{2}}{r_{8} r_{9}} w_{2}, w_{10}=\frac{\rho_{f_{1}}}{r_{10}} w_{7}+\frac{\rho_{f_{2}}}{r_{10}} w_{8}+\frac{\rho_{f_{3}}}{r_{10}} w_{7}, w_{2}=w_{2}>\right.$ 0 , and $v_{1}=0, v_{3}=\left(\frac{\beta_{m} \alpha_{m} \alpha_{f} \psi^{2} \pi_{m} \pi_{f}}{\left(\pi_{m}+\pi_{f}\right)^{2} r_{2} r_{4} r_{7}}+\frac{\beta_{m} \beta_{f} \alpha_{m} \alpha_{f} \psi^{2} \gamma_{f} \pi_{m} \pi_{f}}{\left(\pi_{m}+\pi_{f}\right)^{2} r_{3} r_{4} r_{7} r_{8} r_{9}}+\frac{\beta_{m} \alpha_{m} \alpha_{f} \psi^{2} \gamma_{f} \pi_{m} \pi_{f}}{\left(\pi_{m}+\pi_{f}\right)^{2} r_{3} r_{4} r_{7} r_{8}}+\frac{\alpha_{m} \alpha_{f} \psi^{2} \pi_{m} \pi_{f}}{\left(\pi_{m}+\pi_{f}\right)^{2} r_{3} r_{7}}+\right.$ $\left.\frac{\alpha_{m} \alpha_{f} \psi^{2} \pi_{m} \pi_{f}}{\left(\pi_{m}+\pi_{f}\right)^{2} r_{3} r_{7} r_{8}}+\frac{\beta_{f} \alpha_{m} \alpha_{f} \psi^{2} \gamma_{f} \pi_{m} \pi_{f}}{\left(\pi_{m}+\pi_{f}\right)^{2} r_{3} r_{7} r_{8} r_{9}}\right) v_{2}, v_{4}=\left(\frac{\alpha_{m} \alpha_{f} \psi^{2} \pi_{m} \pi_{f}}{\left(\pi_{m}+\pi_{f}\right)^{2} r_{4} r_{7}}+\frac{\beta_{f} \alpha_{m} \alpha_{f} \psi^{2} \gamma_{f} \pi_{m} \pi_{f}}{\left(\pi_{m}+\pi_{f}\right)^{2} r_{4} r_{7} r_{8} r_{9}}+\frac{\alpha_{m} \alpha_{f} \psi^{2} \gamma_{f} \pi_{m} \pi_{f}}{\left(\pi_{m}+\pi_{f}\right)^{2} r_{4} r_{7} r_{8}}\right) v_{2}, \nu_{5}=0$, $v_{6}=0, v_{7}=\left(\frac{\alpha_{f} \psi \pi_{m}}{\left(\pi_{m}+\pi_{f}\right) r_{7}}+\frac{\beta_{f} \alpha_{f} \psi \gamma_{f} \pi_{m}}{\left(\pi_{m}+\pi_{f}\right) r_{7} r_{8} r_{9}}+\frac{\alpha_{f} \psi \gamma_{f} \pi_{m}}{\left(\pi_{m}+\pi_{f}\right) r_{7} r_{8}}\right) \nu_{2}, v_{8}=\left(\frac{\alpha_{f} \psi \beta_{f} \pi_{m}}{\left(\pi_{m}+\pi_{f}\right) r_{8} r_{9}}+\frac{\alpha_{f} \psi \pi_{m}}{\left(\pi_{m}+\pi_{f}\right) r_{8}}\right) v_{2}, v_{9}=$ $\frac{\alpha_{f} \psi \pi_{m}}{\left(\pi_{m}+\pi_{f}\right) r_{9}} \nu_{2}, v_{10}=0, v_{2}=v_{2}>0$, where $r_{1}=\mu, r_{2}=\left(\gamma_{m}+\mu+\sigma_{m_{1}}\right), r_{3}=\left(\beta_{m}+\mu+\sigma_{m_{2}}\right)$, $r_{4}=\left(\mu+\sigma_{m_{3}}\right), r_{5}=\left(\mu+\varphi_{m}\right), r_{6}=\mu, r_{7}=\left(\gamma_{f}+\mu+\rho_{f_{1}}\right), r_{8}=\left(\gamma_{f}+\mu+\rho_{f_{2}}\right), r_{9}=\left(\mu+\rho_{f_{3}}\right)$, and $r_{10}=\left(\mu+\varphi_{f}\right)$.

Computing the bifurcation coefficients $a$ and $b$ after rigorous simplification yields $a=$ $\frac{v_{2} w_{2} \alpha_{f} \mu}{\pi_{m}+\pi_{f}}+\frac{v_{7} w_{2} \mu \alpha_{m} w_{7}}{\pi_{m}+\pi_{f}}>0, b=\frac{v_{7} w_{2}}{\pi_{m}+\pi_{f}}>0$.

Thus system (2) exhibits backward bifurcation.

\subsection{Sensitivity analysis of the syphilis model}

In this section, we test the effect of system (2) parameters on the basic reproduction number $\mathcal{R}_{0}$ to ascertain the impact of these parameters on syphilis transmission. To get the sensitivity index, we partially differentiated $\mathcal{R}_{0}$ with respect to model (2) parameters. The formula used for the sensitivity analysis in this work, for example, in the case of $\pi_{m}$ is $\frac{\partial \mathcal{R}_{0}}{\partial \pi_{m}} \times \frac{\partial \pi_{m}}{\partial \mathcal{R}_{0}}$, is the same as used for all parameters of model (2). The result is presented in the Table 3.

We observe that the parameters $\pi_{m}, \pi_{f}, \alpha_{m}, \alpha_{f}, \psi, \gamma_{m}$, and $\gamma_{f}$ have positive sensitivity indices, which means that $\mathcal{R}_{0}$ increases with the parameter. The remaining parameters, $\beta_{m}$, $\beta_{f}, \sigma_{m_{1}}, \sigma_{m_{2}}, \rho_{m_{1}}$, and $\rho_{m_{2}}$ have negative values, which implies that $\mathcal{R}_{0}$ decreases for higher values of the parameters. For instance, the implication of the sensitivity index implies an

Table 3 Sensitivity indices of syphilis model $\mathcal{R}_{0}$

\begin{tabular}{lll}
\hline Parameter & Description & Sensitivity Index \\
\hline$\pi_{m}$ & Recruitment rate into susceptible male population & 1 \\
$\pi_{f}$ & Recruitment rate into susceptible female population & 1 \\
$\alpha_{f}$ & Transmission probability of females with syphilis infection & 0.5 \\
$\alpha_{m}$ & Transmission probability of males with syphilis infection & 0.5 \\
$\gamma_{m}$ & Progression rate from male with primary syphilis to male with secondary & 0.042548 \\
& syphilis infection & \\
$\gamma_{f}$ & Progression rate from female with primary syphilis & 0.37906 \\
$\psi$ & Average number of sexual partner per unit time to female with & 1 \\
$\beta_{m}$ & secondary syphilis infection & -0.39089 \\
$\beta_{f}$ & Progression rate from male with secondary syphilis to male with latent & \\
& syphilis infection & -0.0000013015 \\
$\sigma_{m_{1}}$ & Progression rate from female with secondary syphilis to female & \\
$\sigma_{m_{2}}$ & individual with latent syphilis infection & -0.45322 \\
$\rho_{f_{1}}$ & Treatment rate of males with primary stage syphilis & -0.0027407 \\
$\rho_{f_{2}}$ & Treatment rate of males secondary stage syphilis & -0.25536 \\
\hline & Treatment rate females with primary stage syphilis & -0.044735 \\
\hline
\end{tabular}


increase (or decrease) of $\pi_{m}$ by a certain percentage, say, $y \%$ will result in an increase (or decrease) effect on the reproduction number by $y \%$.

\section{Optimal control of the syphilis model}

In this section, we present optimal control interventions for effective management of the syphilis infection. In an attempt to arrest the transmission of syphilis, we incorporated control interventions into system (2) to get

$$
\left\{\begin{array}{l}
\frac{d S_{m}}{d t}=\pi_{m}+\varphi_{m} R_{m}-\alpha_{f} \psi\left(1-u_{1}\right)\left(\frac{I_{f_{p}}+I_{I_{s}}+L_{f}}{N}\right) S_{m}-\mu S_{m} \\
\frac{d I_{m p}}{d t}=\alpha_{f} \psi\left(1-u_{1}\right)\left(\frac{I_{f p}+I_{f_{s}}+L_{f}}{N}\right) S_{m}-\gamma_{m} I_{m p}-\mu I_{m p}-\sigma_{m_{1}} u_{2} I_{m p} \\
\frac{d I_{m s}}{d t}=\gamma_{m} I_{m p}-\beta_{m} I_{m s}-\sigma_{m_{2}} u_{2} I_{m s}-\mu I_{m s} \\
\frac{d L_{m}}{d t}=\beta_{m} I_{m s}-\mu L_{m}-\sigma_{m_{3}} u_{2} L_{m} \\
\frac{d R_{m}}{d t}=\sigma_{m_{1}} u_{2} I_{m p}+\sigma_{m_{2}} u_{2} I_{m s}+\sigma_{m_{3}} u_{2} L_{m}-\mu R_{m}-\varphi_{m} R_{m}, \\
\frac{d S_{f}}{d t}=\pi_{f}+\varphi_{f} R_{f}-\alpha_{m} \psi\left(1-u_{1}\right)\left(\frac{I_{m p}+I_{m s}+L_{m}}{N}\right) S_{f}-\mu S_{f} \\
\frac{d I_{f p}}{d t}=\alpha_{f} \psi\left(1-u_{1}\right)\left(\frac{I_{m p}+I_{m s}+L_{m}}{N}\right) S_{f}-\gamma_{f} I_{f} p-\mu I_{f} p-\rho_{f_{1}} u_{3} I_{f p}, \\
\frac{d I_{f s}}{d t}=\gamma_{f} I_{f} p-\beta_{f} I_{f s}-\rho_{f_{2}} u_{3} I_{f_{s}}-\mu I_{f s} \\
\frac{d L_{f}}{d t}=\beta_{f} I_{f_{s}}-\mu L_{f}-\rho_{f_{3}} u_{3} L_{f}, \\
\frac{d R_{f}}{d t}=\rho_{f_{1}} u_{3} I_{f p}+\rho_{f_{2}} u_{3} I_{f s}+\rho_{f_{3}} u_{3} L_{f}-\mu R_{f}-\varphi_{f} R_{f} .
\end{array}\right.
$$

Basically, we present the objective functional $J$ to investigate the optimal level of effort required to control the syphilis infection. We follow the techniques of [22] to formulate the objective functional, which is given by

$$
J(u)=\int_{0}^{t_{f}}\left[\left(A_{1}\left(I_{p}+I_{s}+L\right)+\frac{1}{2}\left(B_{1} u_{1}^{2}+B_{2} u_{2}^{2}+B_{3} u_{3}^{2}\right)\right] d t,\right.
$$

where $I_{p}=\left(I_{m p}+I_{f p}\right), I_{s}=\left(I_{m s}+I_{f s}\right)$, and $L=\left(L_{m}+L_{f}\right)$.

The factor $u_{1}$ is a control function representing prevention from syphilis infection through the use of condom and safe sex activity in both the male and female populations, $u_{2}$ is a control function representing treatment using antibiotic in male population, and $u_{3}$ is a control function representing treatment using antibiotic in female population. The use of condom and safe sex is aimed at reducing the transmission of syphilis from infected to susceptible individuals. $t_{f}$ is the final time, and the coefficients $A_{1}, B_{1}, B_{2}, B_{3}$ are positive weights to balance the factors. The aim is minimizing the number of males and females with primary stage syphilis $I_{p}$, the number of males and females with secondary stage syphilis $I_{s}$, and the number of males and females with latent stage syphilis $L$ while we keep the cost of controls $u_{1}(t), u_{2}(t), u_{3}(t)$ at minimal level. Thus we seek optimal controls $u_{1}^{*}, u_{2}^{*}, u_{3}^{*}$ such that $J\left(u_{1}^{*}, u_{2}^{*}, u_{3}^{*}\right)=\min _{u_{1}, u_{2}, u_{3}}\left\{J\left(u_{1}, u_{2}, u_{3}\right) \ni u_{1}, u_{2}, u_{3} \in U\right\}$, where $U$ is the set of measurable functions from $\left[0, t_{f}\right]$ onto $[0,1]$. The necessary conditions that an optimal control must satisfy were derived from Pontryagin's maximum principle [32], and the existence of optimal control was derived from the adjoint variable of the state variables satisfying the following set of differential equations. This principle converts system (18) into a problem of minimizing pointwise a Hamiltonian $H$ with respect to $\left(u_{1}, u_{2}, u_{3}\right)$. The 
Hamiltonian is

$$
\begin{aligned}
H= & A_{1}\left(I_{p}+I_{s}+L\right)+\frac{1}{2}\left(B_{1} u_{1}^{2}+B_{2} u_{2}^{2}+B_{3} u_{3}^{2}\right) \\
& +\lambda_{S_{m}}\left[\pi_{m}+\varphi_{m} R_{m}-\left(1-u_{1}\right) \alpha_{f} \psi\left(\frac{I_{f p}+I_{f s}+L_{f}}{N}\right) S_{m}-\mu S_{m}\right] \\
& +\lambda_{I_{m p}}\left[\left(1-u_{1}\right) \alpha_{f} \psi\left(\frac{I_{f p}+I_{f s}+L_{f}}{N}\right) S_{m}-\left(\gamma_{m}+\mu+\sigma_{m_{1}} u_{2}\right) I_{m p}\right] \\
& +\lambda_{I_{m s}}\left[\gamma_{m} I_{m p}-\left(\beta_{m}+\sigma_{m_{2}} u_{2}+\mu\right) I_{m s}\right] \\
& +\lambda_{L_{m}}\left[\beta_{m} I_{m s}-\left(\mu+\sigma_{m_{3}} u_{2}\right) L_{m}\right] \\
& +\lambda_{R_{m}}\left[\sigma_{m 1} u_{2} I_{m p}+\sigma_{m_{2}} u_{2} I_{m s}+\sigma_{m 3} u_{2} L_{m}-\left(\mu+\varphi_{m}\right) R_{m}\right] \\
& +\lambda_{S_{f}}\left[\pi_{f}+\varphi_{f} R_{f}-\left(1-u_{1}\right) \alpha_{m} \psi\left(\frac{I_{m p}+I_{m s}+L_{m}}{N}\right) S_{f}-\mu S_{f}\right] \\
& +\lambda_{I_{f p}}\left[\left(1-u_{1}\right) \alpha_{m} \psi\left(\frac{I_{m p}+I_{m s}+L_{m}}{N}\right) S_{f}-\left(\gamma_{f}+\mu+\rho_{f_{1}} u_{3}\right) I_{f p}\right] \\
& +\lambda_{I_{f s}}\left[\gamma_{f} I_{f p}-\left(\beta_{f}+\rho_{f_{2}} u_{3}+\mu\right) I_{f s}\right] \\
& +\lambda_{L_{f}}\left[\beta_{f} I_{f s}-\left(\mu+\rho_{f_{3}} u_{3}\right) L_{f}\right] \\
& +\lambda_{R_{f}}\left[\rho_{f_{1}} u_{3} I_{f p}+\rho_{f_{2}} u_{3} I_{f s}+\rho_{f_{3}} u_{3} L_{f}-\left(\mu+\varphi_{f}\right) R_{f}\right]
\end{aligned}
$$

where $\lambda_{S_{m}}, \lambda_{I_{m p}}, \lambda_{I_{m s}}, \lambda_{L_{m}}, \lambda_{R_{m}}, \lambda_{S_{f}}, \lambda_{I_{f p}}, \lambda_{I_{f s}}, \lambda_{L_{f}}$, and $\lambda_{R_{f}}$ are the adjoint variables.

Theorem 4 Let $u_{1}^{*}, u_{2}^{*}$, $u_{3}^{*}$ be optimal controls, and let $S_{m}, I_{m p}, I_{m s}, L_{m}, R_{m}, S_{f}, I_{f p}, I_{f s}, L_{f}$, and $R_{f}$ be the solutions of the optimal control problem (18)-(19) that minimize $J\left(u_{1}, u_{2}, u_{3}\right)$ over $U$. Then there exist adjoint variables $\lambda_{S_{m}}, \lambda_{I_{m p}}, \lambda_{I_{m s}}, \lambda_{L_{m}}, \lambda_{R_{m}}, \lambda_{S_{m}}, \lambda_{I_{m p}}, \lambda_{I_{m s}}, \lambda_{L_{m}}, \lambda_{R_{m}}$ satisfying

$$
-\frac{d \lambda_{i}}{d t}=\frac{\partial H}{\partial i}
$$

where $S_{m}, I_{m p}, I_{m s}, L_{m}, R_{m}, S_{f}, I_{f p}, I_{f s}, L_{f}$, and $R_{f}$ are the adjoint variables, and the controls $u_{1}^{*}, u_{2}^{*}, u_{3}^{*}$ obey the optimality conditions

$$
\begin{aligned}
u_{1}^{*}= & \max \{0, \min (1, \\
& \left.\left.\frac{\frac{\alpha_{f} \psi S_{m}^{*}}{N^{*}}\left(I_{f p}^{*}+I_{f s}^{*}+L_{f}^{*}\right)\left(\lambda_{I_{m p}}-\lambda_{S_{m}}\right)+\frac{\alpha_{f} \psi S_{f}^{*}}{N^{*}}\left(I_{m p}^{*}+I_{m s}^{*}+L_{m}^{*}\right)\left(\lambda_{I_{f p}}-\lambda_{S_{f}}\right)}{B_{1}}\right)\right\}, \\
u_{2}^{*}= & \max \{0, \min (1, \\
& \left.\left.\frac{\sigma_{m_{1}} I_{m p}^{*}\left(\lambda_{R_{m}}-\lambda_{I_{m p}}\right)+\sigma_{m_{2}} I_{m s}^{*}\left(\lambda_{R_{m}}-\lambda_{I_{m s}}\right)+\sigma_{m_{3}} L_{m}^{*}\left(\lambda_{R_{m}}-\lambda_{L_{m}}\right)}{B_{2}}\right)\right\}, \\
u_{3}^{*}= & \max \left\{0, \min \left(1, \frac{\rho_{f_{1}} I_{f p}^{*}\left(\lambda_{R_{f}}-\lambda_{I_{f p}}\right)+\rho_{f_{2}} I_{f s}^{*}\left(\lambda_{R_{f}}-\lambda_{I_{s}}\right)+\rho_{f_{3}} L_{f}^{*}\left(\lambda_{R_{f}}-\lambda_{L_{f}}\right)}{B_{3}}\right)\right\} .
\end{aligned}
$$


Proof To prove the theorem, we assessed the differentiated Hamiltonian functional at the optimal control to get the differentiable equations governing the adjoint variables. Hence

$$
\begin{aligned}
& \frac{d \lambda_{S_{m}}}{d t}=\left(1-u_{1}\right) \frac{\alpha_{f} \psi\left(I_{f p}^{*}+I_{f_{s}}^{*}+L_{f}^{*}\right)}{N^{*}}\left(\lambda_{S_{m}}-\lambda_{I_{m p}}\right)+\mu \lambda_{S_{m}}, \\
& \frac{d \lambda_{I_{m p}}}{d t}=\left(\lambda_{I_{m p}}-\lambda_{I_{m s}}\right) \gamma_{m}+\mu \lambda_{I_{m p}}+\sigma_{m_{1}} u_{2}\left(\lambda_{I_{m p}}-\lambda_{R_{m}}\right) \\
& +\frac{\alpha_{m} \psi\left(1-u_{1}\right) S_{f}^{*}}{N^{*}}\left(\lambda_{S_{f}}-\lambda_{I_{f p}}\right)-A_{1} \\
& \frac{d \lambda_{I_{m s}}}{d t}=\left(\lambda_{I_{m s}}-\lambda_{L_{m}}\right) \beta_{m}+\sigma_{m_{2}} u_{2}\left(\lambda_{I_{m s}}-\lambda_{R_{m}}\right)+\mu \lambda_{I_{m s}} \\
& +\frac{\alpha_{m} \psi\left(1-u_{1}\right) S_{f}^{*}}{N^{*}}\left(\lambda_{S_{f}}-\lambda_{I_{f p}}\right)-A_{1}, \\
& \frac{d \lambda_{L_{m}}}{d t}=\sigma_{m_{3}} u_{2}\left(\lambda_{L_{m}}-\lambda_{R_{m}}\right)+\mu \lambda_{L_{m}}+\frac{\alpha_{m} \psi\left(1-u_{1}\right) S_{f}^{*}}{N^{*}}\left(\lambda_{S_{f}}-\lambda_{I_{f p}}\right)-A_{1}, \\
& \frac{d \lambda_{R_{m}}}{d t}=\left(\lambda_{R_{m}}-\lambda_{S_{m}}\right) \varphi_{m}+\mu \lambda_{R_{m}} \\
& \frac{d \lambda_{S_{f}}}{d t}=\left(1-u_{1}\right) \frac{\alpha_{m} \psi\left(I_{m p}^{*}+I_{m s}^{*}+L_{m}^{*}\right)}{N^{*}}\left(\lambda_{S_{f}}-\lambda_{I_{f p}}\right)+\mu \lambda_{S_{f}} \\
& \frac{d \lambda_{I_{f p}}}{d t}=\frac{\alpha_{f} \psi\left(1-u_{1}\right) S_{m}^{*}}{N^{*}}\left(\lambda_{S_{m}}-\lambda_{I_{m p}}\right)+\left(\lambda_{I_{f p}}-\lambda_{I_{f s}}\right) \gamma_{f}+\rho_{f_{1}} u_{3}\left(\lambda_{I_{f p}}-\lambda_{R_{f}}\right)+\mu \lambda_{I_{f p}}-A_{1} \text {, } \\
& \frac{d \lambda_{I_{f s}}}{d t}=\frac{\alpha_{f} \psi\left(1-u_{1}\right) S_{m}^{*}}{N^{*}}\left(\lambda_{S_{m}}-\lambda_{I_{m p}}\right)+\left(\lambda_{I_{f s}}-\lambda_{L_{f}}\right) \beta_{f}+\rho_{f_{2}} u_{3}\left(\lambda_{I_{f s}}-\lambda_{R_{f}}\right)+\mu \lambda_{I_{f s}}-A_{1}, \\
& \frac{d \lambda_{L_{f}}}{d t}=\frac{\alpha_{f} \psi\left(1-u_{1}\right) S_{m}^{*}}{N^{*}}\left(\lambda_{S_{m}}-\lambda_{I_{m p}}\right)+\rho_{f_{3}} u_{3}\left(\lambda_{L_{f}}-\lambda R_{f}\right)+\mu \lambda_{L_{f}}-A_{1}, \\
& \frac{d \lambda_{R_{f}}}{d t}=\left(\lambda_{R_{f}}-\lambda_{S_{f}}\right) \varphi_{f}+\mu \lambda_{R_{f}}
\end{aligned}
$$

with transversality conditions:

$$
\left\{\begin{aligned}
\lambda_{S_{m}}\left(t_{f}\right) & =\lambda_{I_{m p}}\left(t_{f}\right)=\lambda_{I_{m s}}\left(t_{f}\right)=\lambda_{L_{m}}\left(t_{f}\right)=\lambda_{R_{m}}\left(t_{f}\right)=\lambda_{S_{f}}\left(t_{f}\right) \\
& =\lambda_{I_{f p}}\left(t_{f}\right)=\lambda_{I_{f s}}\left(t_{f}\right)=\lambda_{L_{f}}\left(t_{f}\right)=\lambda_{R_{f}}=0
\end{aligned}\right.
$$

Also, the optimal functions $u_{1}^{*}, u_{2}^{*}$, and $u_{3}^{*}$ satisfy

$$
\frac{\partial H}{\partial u_{i}^{*}}=0, \quad i=1,2,3
$$

Therefore

$$
\begin{aligned}
& u_{1}^{*}=\frac{\frac{\alpha_{f} \psi S_{m}^{*}}{N^{*}}\left(I_{f p}^{*}+I_{f s}^{*}+L_{f}^{*}\right)\left(\lambda_{I_{m p}}-\lambda_{S_{m}}\right)+\frac{\alpha_{m} \psi S_{f}}{N^{*}}\left(I_{m p}^{*}+I_{m s}^{*}+L_{m}^{*}\right)\left(\lambda_{I_{f p}}-\lambda_{S_{f}}\right)}{B_{1}}, \\
& u_{2}^{*}=\frac{\sigma_{m_{1}} I_{m p}^{*}\left(\lambda_{R_{m}}-\lambda_{I_{m p}}\right)+\sigma_{m_{2}} I_{m s}^{*}\left(\lambda_{R_{m}}-\lambda_{I_{m s}}\right)+\sigma_{m_{3}} L_{m}^{*}\left(\lambda_{R_{m}}-\lambda_{L_{m}}\right)}{B_{2}}, \\
& u_{3}^{*}=\frac{\rho_{f_{1}} I_{f p}^{*}\left(\lambda_{R_{f}}-\lambda_{I_{f p}}\right)+\rho_{f_{2}} I_{f s}^{*}\left(\lambda_{R_{f}}-\lambda_{I_{f s}}\right)+\rho_{f_{3}} L_{f}^{*}\left(\lambda_{R_{f}}-\lambda_{L_{f}}\right)}{B_{3}} .
\end{aligned}
$$


Table 4 Initial values for the variables

\begin{tabular}{lrl}
\hline Parameters & \multicolumn{1}{c}{ Value } & Source \\
\hline$S_{m}(0)$ & 10,230 & Assumed \\
$I_{m p}(0)$ & 7048 & {$[10]$} \\
$I_{m s}(0)$ & 6067 & {$[10]$} \\
$L_{m}(0)$ & 2600 & Assumed \\
$R_{m}(0)$ & 0 & {$[28]$} \\
$S_{f}(0)$ & 10,960 & Assumed \\
$I_{f p}(0)$ & 7998 & {$[10]$} \\
$I_{f s}(0)$ & 4113 & {$[10]$} \\
$L_{f}(0)$ & 2416 & Assumed \\
$R_{f}(0)$ & 0 & {$[28]$} \\
\hline
\end{tabular}

In accordance with [22], based on typical control arguments involving the bound on the controls, we conclude that as a result of a priori boundedness of the state system and the adjoint system, we obtained the uniqueness of the optimality system (23)-(24). There is a restriction on the length of time interval $\left[0, t_{f}\right]$ so that we can guarantee the uniqueness of the optimality system [27].

\section{Numerical simulations}

In this section, we investigate the impact of interventions on the transmission of syphilis in a population. The optimal control problem (18)-(19) is solved numerically following the techniques in [20], which uses the forward and backward Range-Kutta scheme. We use the variables and parameter values in Tables 2 and 4 to minimize the number of syphilis infections in both males and females. We implement the time level to be five years. The results are presented in Figs. 2-5 using the following strategies:

I. Strategy A: use of condom + treatment of male with syphilis infection.

II. Strategy B: use of condom + treatment of female with syphilis infection.

III. Strategy C: treatment of male with syphilis infection + treatment of female with syphilis infection.

IV. Strategy D: use of condom + treatment of male with syphilis infection + treatment of female with syphilis infection.

\subsection{Strategy A: use of condom + treatment of male with syphilis infection}

In strategy A, we present the simulation of optimal control system (18) with condom use $\left(u_{1}\right)$ as personal protection against infection and treatment of males with syphilis infection $\left(u_{2}\right)$ are implemented, whereas treatment of female with syphilis infection $\left(u_{3}\right)$ is set to zero. Figures $2(b-d)$ show the results of the implementation of strategy A. There is a significant difference in the population of males with primary syphilis infection $I_{m p}$, males with secondary syphilis infection $I_{m s}$, and males with latent syphilis infection $L_{m}$ when optimal use of condom $\left(u_{1} \neq 0\right)$ and treatment of males with syphilis infection $\left(u_{2} \neq 0\right)$ were compared to the population without optimal control strategy. Figures $2(\mathrm{~g}-\mathrm{i})$ show the results for females with primary syphilis infection $I_{m p}$, females with secondary syphilis infection $I_{m s}$, and females with latent syphilis infection $L_{m}$ when strategy A is applied. The population of females with primary syphilis infection $I_{f p}$, females with secondary syphilis infection $I_{f s}$, and females with latent syphilis infection $L_{f}$ drop when optimal use of con$\operatorname{dom}\left(u_{1} \neq 0\right)$ and treatment of males with syphilis infection $\left(u_{2} \neq 0\right)$ against the population without optimal control strategy. Figure $2(\mathrm{k})$ depicts the control profile for strategy A. We 


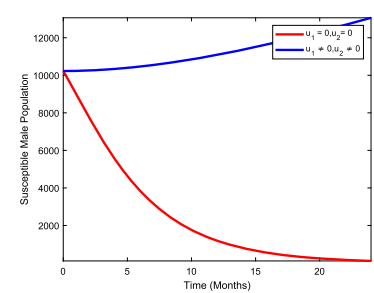

(a)

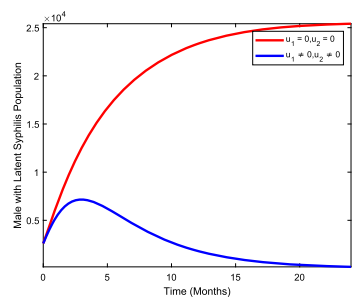

(d)

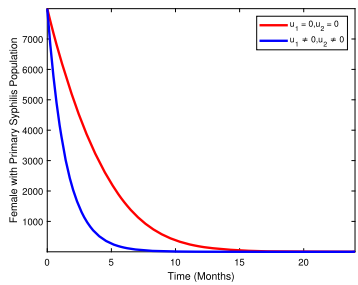

(g)

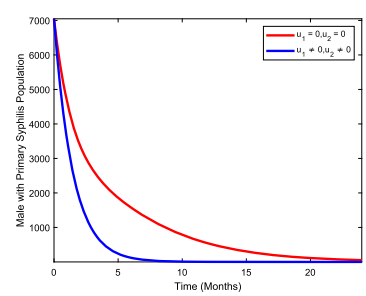

(b)

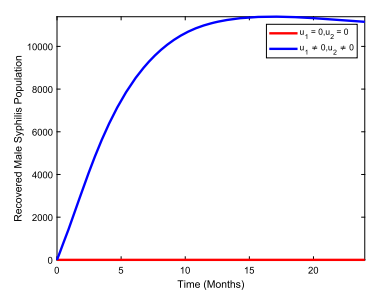

(e)

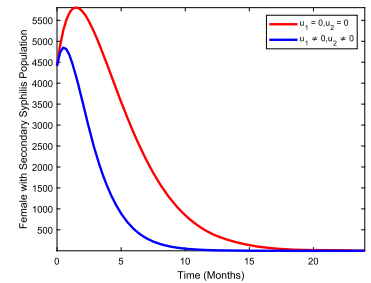

(h)

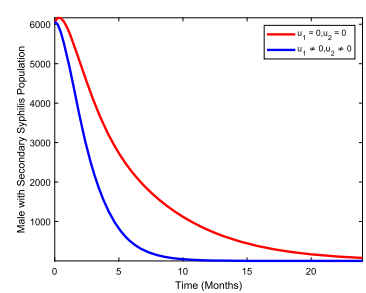

(c)

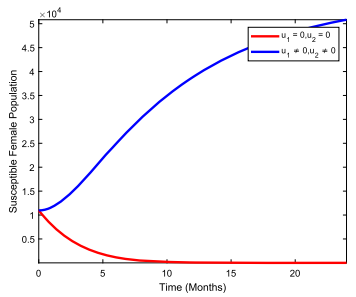

(f)

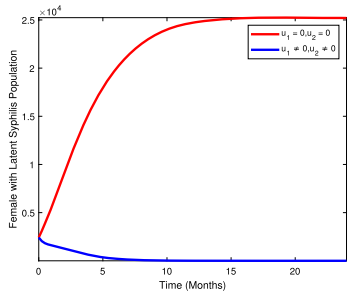

(i)

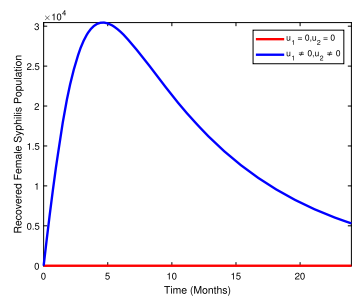

(j)

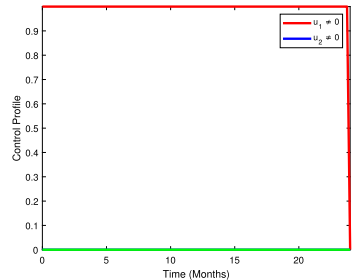

(k)

Figure 2 Use of condom + treatment of male with syphilis infection

observed that the control curve for the use of condoms remains at the upper bound for almost the entire duration of the study period, that is, 24 months, whereas the curve for the treatment of males with syphilis infection remains at a lower bound for the duration of the study.

\subsection{Strategy B: use of condom + treatment of female with syphilis infection}

This strategy shows the simulation of optimal control system (18) for condom use $\left(u_{1}\right)$ as personal protection against infection and treatment of females with syphilis infection $\left(u_{3}\right)$ while treatment of male with syphilis infection $\left(u_{2}\right)$ is fixed at zero. Figures $3(\mathrm{~b}-\mathrm{d})$ show that there is a clear change in the population of males with primary syphilis infection $I_{m p}$, males with secondary syphilis infection $I_{m s}$, and males with latent syphilis infection $L_{m}$ when strategy $B$ is effected compared to the population without optimal control strategy $B$. Figures $3(\mathrm{~g}-\mathrm{i})$ show the results for females with primary syphilis infection $I_{f p}$, females with 


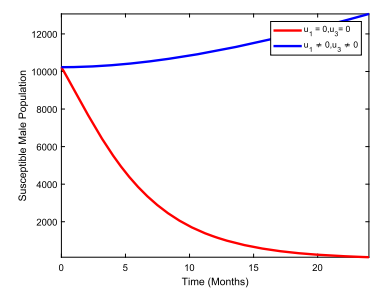

(a)



(d)

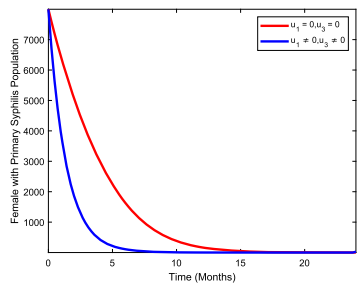

(g)

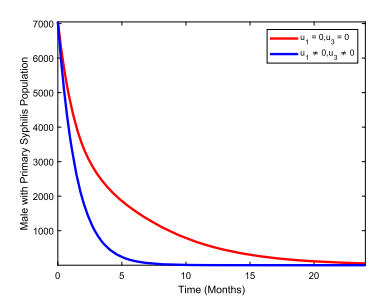

(b)

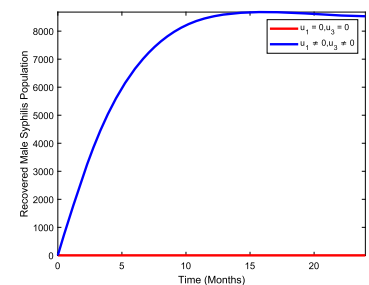

(e)

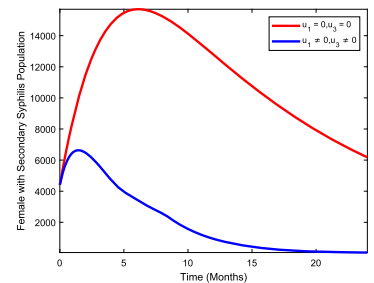

(h)

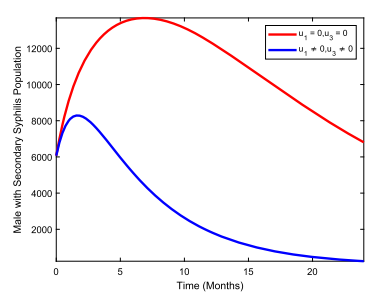

(c)

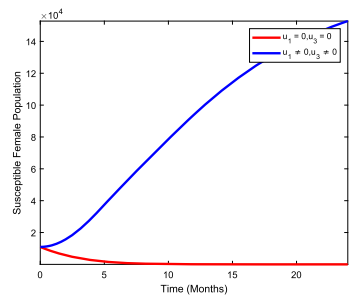

(f)

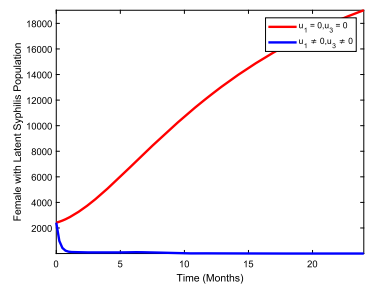

(i)

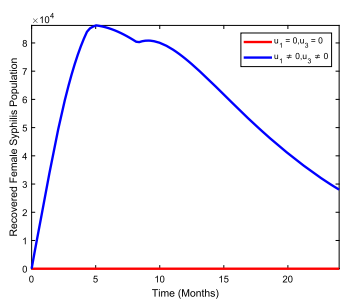

(j)

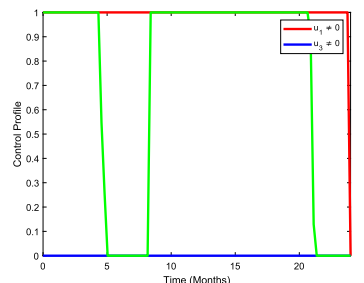

(k)

Figure 3 Use of condom + treatment of female with syphilis infection

secondary syphilis infection $I_{f s}$, and females with latent syphilis infection $L_{f}$ when strategy $B$ is applied. We observed that the population of females with primary syphilis infection $I_{f p}$, females with secondary syphilis infection $I_{f s}$ and females with latent syphilis infection $L_{f}$ reduce when optimal use of condom $\left(u_{1} \neq 0\right)$ and treatment of males with syphilis infection $\left(u_{3} \neq 0\right)$ are implemented when compared to the case without control strategy. Figure 3(k) shows the control profile for strategy B. From the control curve we observed that the use of condoms remains at the upper bound for almost the entire duration of the study period, that is, 24 months, whereas the curve for the treatment of females with syphilis infection is unstable for the entire period of implementation of the strategy.

\subsection{Strategy C: treatment of male and female with syphilis infection}

In strategy $\mathrm{C}$, we use treatment of males with syphilis infection $\left(u_{2}\right)$ and treatment of female with syphilis infection $\left(u_{3}\right)$ to optimize the objective functional (19) while the use of 


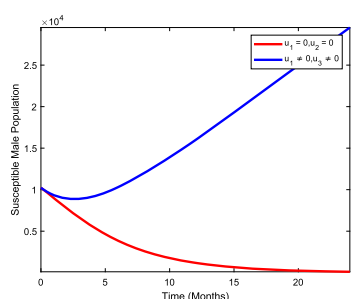

(a)

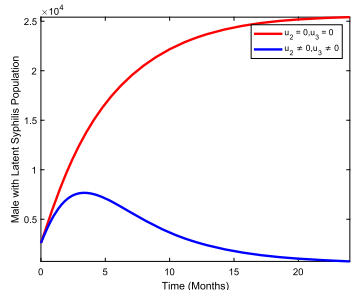

(d)

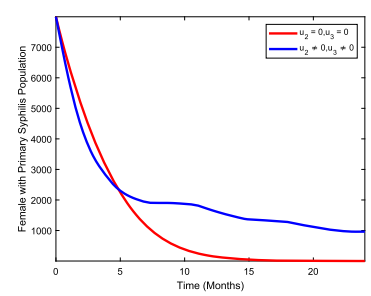

(g)

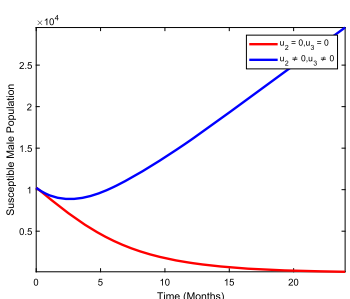

(b)

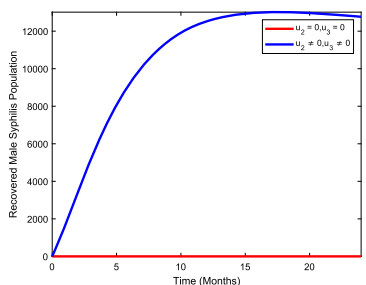

(e)

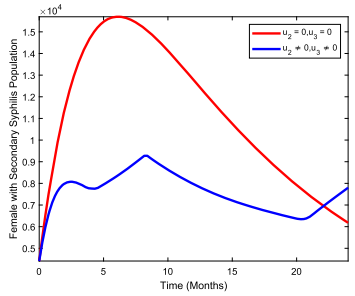

(h)

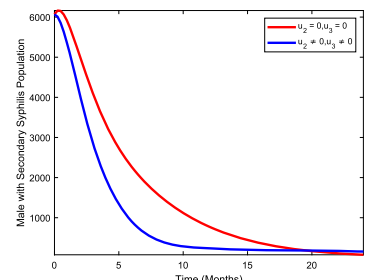

(c)

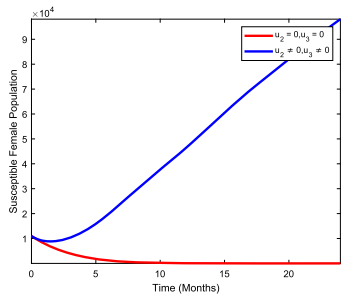

(f)

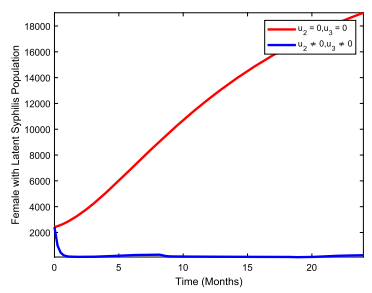

(i)

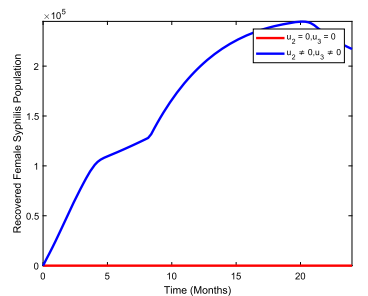

(j)

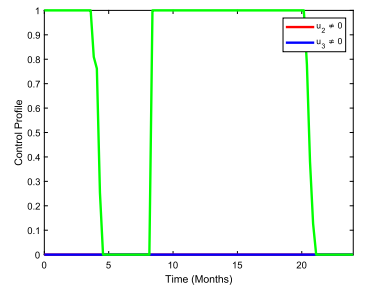

(k)

Figure 4 Treatment of male with syphilis infection + treatment of female with syphilis infection

condom $\left(u_{1}\right)$ is set at zero. Figures $4(\mathrm{~b}-\mathrm{d})$ show that there is a drastic change in the population of males with primary syphilis infection $I_{m p}$, males with secondary syphilis infection $I_{m s}$, and males with latent syphilis infection $L_{m}$ when strategy $\mathrm{C}$ is effected compared to the population without optimal control strategy C. Figures 4(g-i) depict the results for females with primary syphilis infection $I_{f p}$, females with secondary syphilis infection $I_{f s}$, and females with latent syphilis infection $L_{f}$ when strategy B is applied. We observed that the population of females with primary syphilis infection $I_{f p}$, females with secondary syphilis infection $I_{f}$, and females with latent syphilis infection $L_{f}$ diminishes when optimal treatment of males with syphilis infection $\left(u_{2} \neq 0\right)$ and treatment of females with syphilis infection $\left(u_{3} \neq 0\right)$ are implemented when compared to the case without control. Figure $4(\mathrm{k})$ shows the control profile for strategy $\mathrm{C}$. We observed that the treatment of males with syphilis stays at a lower bound throughout the duration of the study period, whereas the 


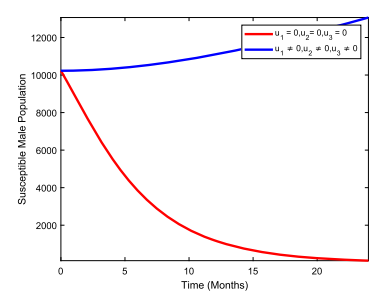

(a)

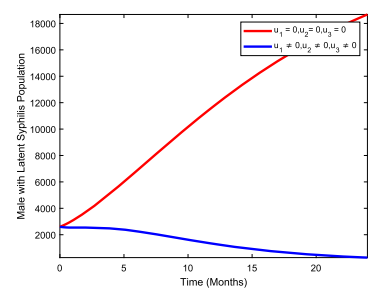

(d)

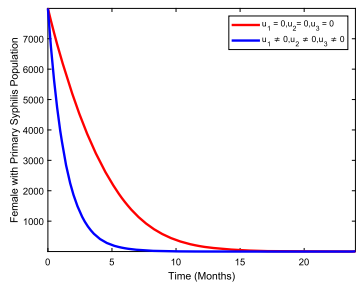

(g)

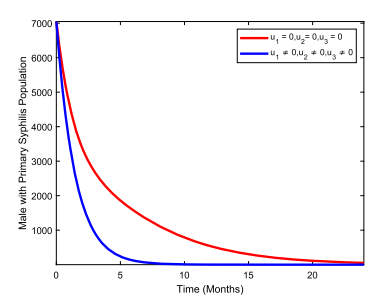

(b)

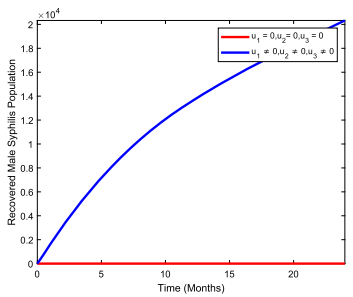

(e)

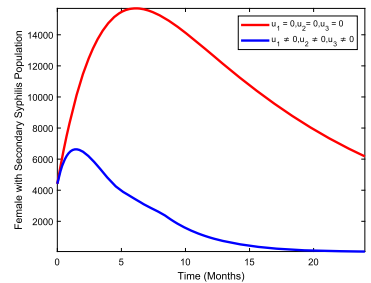

(h)

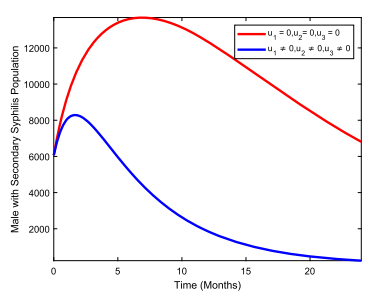

(c)

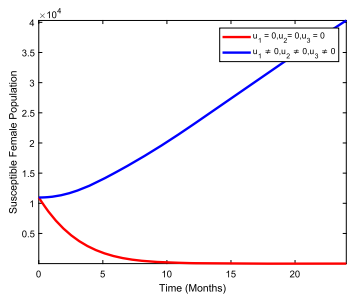

(f)

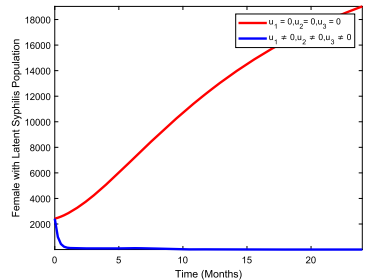

(i)

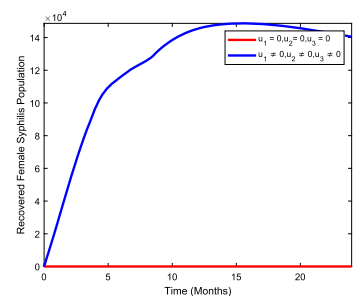

(j)

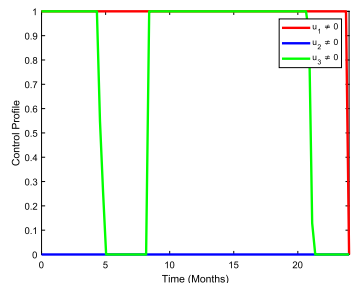

(k)

Figure 5 Use of condom + treatment of male with syphilis infection + treatment of female with syphilis infection

curve for the treatment of females with syphilis infection oscillates throughout the period of implementation of the strategy.

\subsection{Strategy D: use of condom + treatment of male with syphilis infection + treatment of female with syphilis infection}

Strategy D presents use of condom $\left(u_{1}\right)$, treatment of males with syphilis infection $\left(u_{2}\right)$, and treatment of female with syphilis infection $\left(u_{3}\right)$ to optimize the objective functional (19). Figures 5(b-d) depict a more significant decrease in the population of males with primary syphilis infection $I_{m p}$, males with secondary syphilis infection $I_{m s}$, and males with latent syphilis infection $L_{m}$ when strategy $C$ is effected compared to the population without optimal control strategy $C$. We also compared strategy D to other strategies and found out that the strategy shows a significant decline in the number of the infected population compared to other strategies. Figures $5(\mathrm{~g}-\mathrm{i})$ depict the results for females with primary 
syphilis infection $I_{f p}$, females with secondary syphilis infection $I_{f s}$, and females with latent syphilis infection $L_{f}$ when strategy $\mathrm{C}$ is implemented. We observed that the population of females with primary syphilis infection $I_{f p}$, females with secondary syphilis infection $I_{f s}$, and females with latent syphilis infection $L_{f}$ diminishes when optimal use of condom $\left(u_{1} \neq 0\right)$, treatment of males with syphilis infection $\left(u_{2} \neq 0\right)$, and treatment of females with syphilis infection $\left(u_{3} \neq 0\right)$ are implemented when compared to the case without control. Figure 5(k) depicts the control profile for strategy D. We observed that the curve for use of condom is at the upper bound for almost the entire period of the study (24 months), treatment of males with syphilis stays at lower bound throughout the duration of the study period, whereas the curve for the treatment of females with syphilis infection oscillates throughout the period of implementation of the strategy.

However, we realized that each of the four strategies explored in this study has positively demonstrated that the spread of syphilis can be halted once the desired group is targeted with given proper treatment. We equally noted that treating males can have an effect on females with syphilis infection and vice versa. Of the four strategies, strategy D, condom plus treatment in both the male and the female population, yielded the best result when compared to the use of treatment only strategy or condom plus treatment in male alone or condom plus treatment in female only strategies. This is supported by [21, 30,37], respectively; treating the syphilis disease in its primary level and directing resources to the use of condoms plus treatment in a population would widely and immensely contribute in controlling the spread of syphilis.

\section{Conclusion}

In this research, we proposed and studied a mathematical sex-structured syphilis model with three stages of infection and three control strategies, and loss of immunity. We assumed a constant control for the control parameters in the analytical solution. The positivity of the solution was proved, and the system of nonlinear differential equations is found to be biologically and mathematically well-posed. We obtained the basic reproduction number using the next-generation method. The syphilis-free and syphilis-present equilibria were established. The syphilis-free equilibrium is locally asymptotically stable when $\mathcal{R}_{0}<1$ and unstable when $\mathcal{R}_{0}>1$. The global stability of syphilis-free equilibrium is proved to be globally asymptotically stable when the associated reproduction number $\mathcal{R}_{0}<1$. This implies that the disease will completely die out in a stable equilibrium, whereas it will persist and become endemic in an unstable equilibrium. We used Pontryagin's maximum principle to investigate the optimal level required to curtail the spread of syphilis in a population. Numerical results show that the best strategy for control of syphilis transmission is strategy $\mathrm{D}$, the combination of condom usage for the prevention and treatment of infected male and female population.

Acknowledgements

The authors acknowledge the facilities provided by Modibbo Adama University of Technology, Yola, Nigeria, and Tertiary Education Trust Fund.

Funding

The research received no external funding. 


\section{Competing interests}

The authors declare that they have no competing interests.

\section{Authors' contributions}

The development and simulation of the model was done by AAM. Model analysis, discussion of results,and typesetting were implemented by YB, DJW, and DD. All authors approved the final manuscript.

\section{Author details}

${ }^{1}$ Department of Mathematics, Modibbo Adama University of Technology, Yola, Nigeria. ${ }^{2}$ Cheikh Anta Diop University, Dakar, Senegal.

\section{Publisher's Note}

Springer Nature remains neutral with regard to jurisdictional claims in published maps and institutional affiliations.

Received: 20 November 2020 Accepted: 24 May 2021 Published online: 11 June 2021

\section{References}

1. Abdullahi, A.A.: On optimal strategy for the control of syphilis disease. NAMP 3, 119-122 (2017)

2. Andrawus, J., Eguda, F.Y.: Mathematical analysis of a model for syphilis endemicity. Int. J. Sci. Eng. Appl. Sci. 3(8), 48-72 (2017)

3. Araz, S.I: Analysis of a Covid-19 model: optimal control, stability and simulations. Alex. Eng. J. 60, 647-658 (2021)

4. Atangana, A., Araz, S.I.: Nonlinear equations with global differential and integral operators: existence, uniqueness with application to epidemiology. Results Phys. 20, 103593 (2021)

5. Atangana, E., Atangana, A.: Face masks simple but powerful weapons to protect against COVID-19 spread: can they have side effects? Results Phys. 19, 103425 (2020)

6. Bulut, H., Kumar, D., Singh, R., Swoop, R., Baskonus, H.M.: Analytic study for fractional model of HIV infection of CD4 ${ }^{+} T$ lymphocyte cells. Math. Nat. Sci. 2, 33-43 (2018)

7. Buonomo, B., Lactignola, D.: Analysis of a tuberculosis model with a case study in Uganda. J. Biol. Dyn. 4(6), 571-593 (2010)

8. Castillo-Chavez, C., Feng, Z., Huang, W.: On the computation of $R_{0}$ and its role on global stability. IMA Vol. Math. Appl. $125,229(2002)$

9. Castillo-Chavez, C., Song, B.: Dynamical models of tuberculosis and their applications. Math. Biosci. Eng. 1(2), 361-404 (2004)

10. Centre for Disease Control and Prevention: sexually transmitted disease surveillance. Retrieved on 5th October, 2019 from www.cdc.gov/std/stats17/syphilis.htm (2017)

11. Centre for Disease Control and Prevention: Sexually transmitted diseases treatment guidelines. US department of health and human services. Morb. Mort. Wkly. Rep. 64(3), 34-36 (2015)

12. Clement, M.E., Okeke, N.L., Hicks, C.B.: Treatment of syphilis: a systematic review. JAMA 312(18), 1905-1917 (2014)

13. Fenton, K.A., Breban, R., Vardavas, R., et al.: Infectious syphilis in high-income settings in the 21st century. Lancet Infect. Dis. 8(4), 244-253 (2008). https://doi.org/10.1016/s1473-3099(08)70065.3

14. French, P. Syphilis. BMJ, Br. Med. J. 334(7585), 143-147 (2007). https://doi.org/10.1136/bmj.39085.518148

15. Garnett, G.P., Aral, S.O., Hoyle, D.V., Cates, W., Anderson, R.M.: The natural history of syphilis. Implications for the transmission dynamics and control of infection. J. Sex. Transm. Dis. 24(4), 185-200 (1997). Accessed 15th January, 2016, retrieved on 12th February, 2019 from https://www.researchgate.net/publication/14115022

16. Guckenheimer, J., Holmes, P.: Nonlinear oscillations, dynamical systems and bifurcations of vector fields. J. Appl. Mech. 51(4), 947 (1984)

17. Iboi, E., Okuonghae, D.: Population dynamics of a mathematical model for syphilis. Appl. Math. Model. 40, 3573-3590 (2016)

18. Kilmarx, P.H., St Louis, M.E.: Epidemiology research branch division of STD/HIV prevention: center for disease control and prevention. Am. J. Publ. Health 85(8), 1053-1054 (1995)

19. Kimbir, A.R., Oduwole, H.K.: A mathematical model of HIV/AIDS transmission dynamics considering counseling and antiretroviral therapy. J. Mod. Math. Stat. 2(5), 166-169 (2008)

20. Lenhart, S.M., Workman, J.T.: Optimal Control Applied to Biological Model, vol. 15. CRC Press, New York (2007)

21. Melrose, P.: Nigeria prevalence of syphilis (percentage of women attending antenatal care). Retrieved on 12th July 2019 from https://tradingeconomics.com (2019)

22. Michael, M., Libin, M., Weimin, H.: Convergence of the forward-backward sweep method in optimal control. Comput. Optim. Appl. 53(1), 207-226 (2012)

23. Milner, F., Zhao, R.: A new mathematical model of syphilis. Math. Model. Nat. Phenom. 5(6), 96-108 (2010). https://doi.org/10.1051/mmnp/20105605

24. Muthuri, G.G., Malonza, D.M.: Mathematical modeling of TB-HIV CO infection, case study of tigania West sub country, Kenya. J. Adv. Math. Comput. Sci. 27(5), 1-18 (2018)

25. Naik, P.A., Yavuz, M., Qureshi, S., Zu, J., Townley, S.: Modeling and analysis of COVID-19 epidemics with treatment in fractional derivatives using real data from Pakistan. Eur. Phys. J. Plus 135, 795 (2020)

26. Nwanko, A., Okuonghae, D.: Mathematical analysis of the transmission dynamics of HIV syphilis co-infection in the presence of treatment for syphilis. Bull. Math. Biol. 80(3), 437-492 (2018). https://doi.org/10.1007/s11538-017-0384-0

27. Okosun, K.O., Ouifki, R., Marcus, N.: Optimal control analysis of a malaria disease transmission model that includes treatment and vaccination with waning immunity. Biosystems 106(2-3), 136-145 (2011)

28. Okuonghae, D., Gumel, A.B., Ikhimwin, B.O., Iboi, E.: Mathematical assessment of the role of early latent infections and targeted control strategies on syphilis transmission dynamic. Acta Biotheor. 67(1) 47-84 (2019). https://doi.org/10.1007/s10441-018-9336-9 
29. Oyeniyi, R.B., Are, E.B., Ibraheem, M.O.: Mathematical modeling of syphilis in a heterogeneous setting with complications. J. Niger. Math. Soc. 36(3), 479-490 (2017)

30. Peeling, R., Mabey, D., Fitzgerald, D., Watson-Jones, D.: Avoiding HIV and dying of syphilis. Lancet 364, 1561-1563 (2004)

31. Peter, T.A., Kidd, S.: Trends in death due to syphilis; United States 1968-2015. Sex. Transm. Dis. 46(1), 37-40 (2019). https://doi.org/10.1097/OLQ.0000000000000898

32. Pontryaggin, L.S., Boltryanskii, V.G., Gamkrelidze, R.V., Mishchenco, E.F.: Mathematical Theory of Optimal Process, vol. 4. Gordon \& Breach, New York (1986)

33. Prabhakararao, G.: Mathematical modelling of syphilis disease; a case study with reference to Anantapur district-Andhra Pradesh-India. Int. J. Eng. Res. Appl. 4(10), 29-39 (2014)

34. Qureshi, S.: Periodic dynamics of rubella epidemic under standard and fractional Caputo operator with real data from Pakistan. Math. Comput. Simul. (2020). https://doi.org/10.1016/j.matcom.2020.06.002

35. Qureshi, S.: Effects of vaccination on measles dynamics under fractional conformable derivative with Liouville-Caputo operator. Eur. Phys. J. Plus 135, 63 (2020)

36. Qureshi, S., Atangana, A.: Fractal-fractional differentiation for the modeling and mathematical analysis of nonlinear diarrhea transmission dynamics under the use of real data. Chaos Solitons Fractals 136, 109812 (2020)

37. Saad-Roy, C.M., Shuai, Z., Driessche, P.V.: A mathematical model of syphilis transmission in an MSM population. Math Biosci. 277, 59-70 (2016)

38. Selmane, S.: Optimal control applied to resistance strategies for the transmission dynamics of syphilis. University of Science and Technology Houari Boumediene, Algeria (2014)

39. This day Newspaper: Borno with highest cases of syphilis, 24 March 2016 (2016)

40. Tuddenham, S., Ghanem, K.G.: Emerging trends and persistent challenges in the management of adult syphilis. BMC Infect. Dis. 15(351), 1-8 (2015)

41. Van den Driessche, P., Watmough, J.: Reproduction numbers and sub-threshold endemic equilibra for compartmental models of disease transmission. Math. Biosci. 180(1-2), 29-48 (2002)

42. World Bank: Life expectancy at birthdata.worldbank.org. Accessed 5th October, 2019 (2016)

43. World Health Organization: Global incidence and prevalence of selected curable sexually transmitted infections (2012)

44. World Health Organization: Guideline for treatment of treponema pallidum (syphilis). Accessed 11th April, 2019 Retrieved 12th April, 2019 from http://www.who.int/reproductivehealth/publications/rtis/syphilis (2016)

45. Zhou, Y.H., Yang, Y.R., Zhang, H.J.: Stability of non-monotone critical waves in a population dynamics model with spatio-temporal delay. Math. Nat. Sci. 2, 8-23 (2018)

\section{Submit your manuscript to a SpringerOpen ${ }^{\circ}$ journal and benefit from:}

- Convenient online submission

- Rigorous peer review

- Open access: articles freely available online

- High visibility within the field

- Retaining the copyright to your article

Submit your next manuscript at $>$ springeropen.com 